\title{
NGL-2 Is a New Partner of PAR Complex in Axon Differentiation
}

\author{
Gang Xu, ${ }^{1,2,3}$ Rong Wang, ${ }^{2}$ Zeyou Wang, ${ }^{2}$ Qianqian Lei, ${ }^{2}$ Zhibin Yu, ${ }^{2}$ Changhong Liu, ${ }^{2}$ Peiyao Li, ${ }^{2}$ Zengjie Yang, ${ }^{4}$ \\ Xiping Cheng, ${ }^{5}$ Guiyuan $\mathrm{Li},{ }^{1,2}$ and Minghua $\mathrm{Wu}^{2}$ \\ ${ }^{1}$ Hunan Provincial Tumor Hospital and the Affiliated Tumor Hospital of Xiangya Medical School, Central South University, and ${ }^{2}$ School of Basic Medical \\ Science, Cancer Research Institute, Central South University, Changsha 410078, Hunan, People's Republic of China, ${ }^{3}$ Medical College, University of South \\ China, Hengyang 421001, Hunan, People's Republic of China, ${ }^{4}$ Cancer Biology Program, W345, Fox Chase Cancer Center, Philadelphia, Pennsylvania 19111, \\ and ${ }^{5}$ Department of Molecular, Cellular, and Developmental Biology, University of Michigan, Ann Arbor, Michigan 48109
}

Neuronal polarization is pivotal for neural network formation during brain development. Axon differentiation is a hallmark of initial neuronal polarization. Here, we report that the leucine-rich repeat-containing protein netrin-G ligand-2 (NGL-2) as a polarity regulator that localizes asymmetrically in rat hippocampal neurons and is required for differentiation of the future axon. NGL-2 was associated with PAR complex, and this interaction resulted in local stabilization of axonal microtubules. Further study showed that the $\mathrm{C}$ terminal of NGL-2 binds to the PDZ domain of PAR6, and NGL-2 interacts with PAR3 and atypical PKC $\zeta$ (aPKC $\zeta$ ), with PAR6 acting as a bridge or modifier. Then, NGL-2 regulates the local stabilization of microtubules and promotes axon differentiation by the aPKC $\zeta /$ microtubule affinity-regulating kinase 2 pathway. These findings reveal the critical role of NGL-2 in regulating axon differentiation in rat hippocampal neurons and reveal a novel partner of the PAR complex.

Key words: axon differentiation; LRRC4; microtubule stability; neuronal polarity; NGL-2

\section{Introduction}

Axon differentiation is the fundamental process that gives rise to a complex morphology and physiology of neurons (Stiess and Bradke, 2011). In hippocampal neurons, the PAR complex, consisting of PAR3, PAR6, and atypical PKC (aPKC), is required for axon differentiation (Shi et al., 2003; Nishimura et al., 2004). Both PAR3 and PAR6 contain PDZ (PSD-95/Dlg/ZO-1) domains, which may interact with other PDZ domains or with specific sequence motifs (Sheng and Sala, 2001; Shi et al., 2003). PAR3 contains three PDZ domains; the first PDZ domain in PAR3 interacts with the PDZ domain of PAR6, and the PAR3 C-terminal region binds to the kinase domain of aPKC (Lin et al., 2000). PAR6 contains a single PDZ domain, which associates with PAR3 and binds the regulatory domain of aPKC via its $\mathrm{N}$-terminal region (Noda et al., 2001). In this fashion, the combined use of interaction and catalytic domains assembles a complex that can be positioned at specific subcellular sites, and that receives and transmits polarity signals (Wiggin et al., 2005). The

\footnotetext{
Received Nov. 17, 2014; revised March 23, 2015; accepted March 25, 2015

Author contributions: G.X., G.L., and M.W. designed research; G.X., Z.W., Q.L., Z. Yu, C.L., and P.L. performed research; G.X., R.W., and Z.W. contributed unpublished reagents/analytic tools; G.X., R.W., Z.W., and M.W. analyzed data; G.X., Z. Yang, X.C., and M.W. wrote the paper.

This study was supported by the National Science Foundation of China (Grant 31201023), the Open-End Fund for the Valuable and Precision Instruments of Central South University (Grant CSUZC2014041), and the 111 Project (Grant 111-2-12).

Correspondence should be addressed to Minghua Wu, School of Basic Medical Science, Cancer Research Institute, Central South University, Changsha 410078, Hunan, People's Republic of China. E-mail: wuminghua554@aliyun.com.

DOI:10.1523/JNEUROSCI.4726-14.2015

Copyright (C) 2015 the authors $\quad 0270-6474 / 15 / 357153-12 \$ 15.00 / 0$
}

downstream effectors of PAR complex in axon differentiation have been examined. For example, aPKC directly phosphorylates microtubule affinity-regulating kinase 2 (MARK2), in turn leads to the dephosphorylation of tau, and, finally, promotes the assembly of stable microtubulins and axon differentiation (Chen et al., 2006). The PAR complex is also associated with STEF/Tiam and $\mathrm{Cdc} 42$, both of which are required for neuronal polarization (Lin et al., 2000; Nishimura et al., 2005). The extensive interplay between the PAR complex and other proteins suggests that the PAR complex can change components dynamically to modulate the outcome of signaling inputs as well (Aranda et al., 2008).

Netrin-G ligand-2 (NGL-2) is a receptor for netrin-G2, a member of the neuronal cell adhesion molecule. NGL-2 and netrin-G2 bind in an isoform-specific manner, and the C-terminus PDZ binding domain of NGL-2 associates with PSD-95 (Woo et al., 2009). NGL-2 regulates the formation of excitatory synapses through the trans-synaptic netrin-G2-NGL2-PSD-95 adhesion fashion and selectively regulates excitatory transmission at Schaffer collateral synapses in CA1 stratum radiatum of hippocampus (Woo et al., 2009; DeNardo et al., 2012). NGL-2 is also expressed in horizontal cells (HCs) of the retina, and localizes to the synapses between $\mathrm{HC}$ axons and rod photoreceptors, regulating presynaptic and postsynaptic specializations, and adjusting visual signal transmission (Soto et al., 2013). NGL-2 is also named leucine-rich repeat C4 (LRRC4), which plays an important role in the initiation and development of glioma (Wu et al., 2008; Tang et al., 2011,2012). Although NGL-2 has been recognized as a central component of synapse formation in hippocampus and retina, there are still many interesting ques- 
tions that remain to be addressed, including the possible involvement of NGL-2 in axon differentiation of neurons.

Here, we found that the NGL-2 protein is enriched in the axon, especially at the tip of rat hippocampal neurons. NGL-2 plays a critical role in axon differentiation. To achieve this, NGL-2 forms a dynamic complex with PAR3/PAR6/aPKC $\zeta$. NGL-2 is recruited by PAR6 and selectively localizes in future axons. In the presence of PAR6, NGL-2 interacts with aPKC $\zeta$, then stabilizes microtubules to promote axon differentiation through the aPKC $\zeta / M A R K 2$ pathway.

\section{Materials and Methods}

Antibodies and constructs. The antibodies used for immunostaining or immunoblotting were as follows: NGL-2, PAR3, PAR6, aPKC $\zeta$, and phosphorylated (p)-aPKC $\zeta$ from Santa Cruz Biotechnology; Tau-1 from Millipore; MARK2, p-MARK2 (T595), Tau, and p-Tau (S262) from Cell Signaling Technology; and acetylated $\alpha$-tubulin, $\alpha$-tubulin, Tuj1, Flag, Myc, and HA from Sigma-Aldrich. Full-length rat NGL-2 (DQ119102, amino acids 1-652; from a brain cDNA library; Clontech) and NGL$2 \triangle \mathrm{PB}$ (amino acids 1-649) were subcloned into pEGFP-C1 or pCMVFlag (Clontech). PAR3, PAR6, and aPKC $\zeta$ of rat were subcloned into pDsRED-N1, pCMV-Myc, or pCMV-HA (Clontech), respectively. For small interfering RNA (siRNA) knockdown, nucleotides 1488-1506 of rat NGL-2 (5'-GATATCACCTGAGGACATA-3' DQ119102) was subcloned into pSUPER (Clontech). The mutant of NGL-2 (NGL-2M) was subcloned into pCDNA 3.1 (Clontech). Double-stranded oligonucleotides targeting against PAR6 were synthesized by Shanghai GenePharma, siRNA sequences were designed according to nucleotides $738-760$ of rat PAR6 (5'-GCCTGCCAACCAGCGTAATAATG-3'; AY682587.1).

Neuronal culture and electroporation. Hippocampi of embryonic day 18 (E18) rat embryos (those of either sex were used) were digested with $0.125 \%$ trypsin-EDTA for $20 \mathrm{~min}$ at $37^{\circ} \mathrm{C}$, followed by trituration with pipettes in the plating medium (DMEM with $10 \%$ FBS and 10\% F12 medium). Dissociated neurons were transfected by electroporation using the Amaxa nucleofector device. Neurons were plated onto coverslips coated with poly-D-lysine $(0.1 \mathrm{mg} / \mathrm{ml})$. After culturing for $4 \mathrm{~h}$, media were changed into neuronal culture medium (neurobasal media containing $1 \%$ glutamate and 2\% B27 medium).

Immunocytochemistry. Neurons were washed with PBS, fixed in $4 \%$ PFA at room temperature for $30 \mathrm{~min}$, and incubated with $0.1 \%$ Triton X-100 in PBS for 10 min. After blocking with $10 \%$ goat serum in PBS at room temperature for $1 \mathrm{~h}$ or $4^{\circ} \mathrm{C}$ overnight, neurons were incubated in primary antibodies at $4^{\circ} \mathrm{C}$ for $12 \mathrm{~h}$ and subsequently with Alexa Fluor 350-, FITC- or rhodamine-conjugated secondary antibodies. Coverslips were mounted and examined using a Zeiss confocal microscope. Images were taken with AxioVision microscopy software (Zeiss), and were analyzed with AxioVision (Zeiss), ImageJ, and Adobe Photoshop (Adobe).

Immunoprecipitation assay. For the immunoprecipitation assay of endogenous proteins, brains of E18 rat embryos (rats of either sex were used) were extracted by the addition of lysis buffer (20 mM Tris- $\mathrm{HCl}, \mathrm{pH}$ 7.5, 1 mм MEDTA, 150 mм NaCl, 1\% NP-40, $50 \mu \mathrm{g} / \mathrm{ml} \mathrm{PMSF,} 10 \mu \mathrm{g} / \mathrm{ml}$ leupeptin, $2 \mu \mathrm{g} / \mathrm{ml}$ aprotinin, and $1 \mu \mathrm{g} / \mathrm{ml}$ pepstatin) and then clarified by centrifugation at $10,000 \times g$ for $20 \mathrm{~min}$ at $4^{\circ} \mathrm{C}$. For the interaction assay of exogenous NGL-2 and PAR3/PAR6/PKC $\zeta$, HEK293 cells were transfected with the indicated plasmids and extracted by the addition of lysis buffer. The soluble supernatants were incubated with the indicated antibodies for $1 \mathrm{~h}$ at $4^{\circ} \mathrm{C}$. The immunocomplexes were then precipitated with protein A-Sepharose CL-4B (GE Healthcare). The immunocomplexes were washed three times with lysis buffer, eluted by boiling in sample buffer for SDS-PAGE, and then subjected to immunoblot analysis with the indicated antibodies.

Pull-down assay. GST fusion proteins containing various deletions of NGL-2 cytoplasmic domain or deletions of PAR6 were expressed in BL21 (DE3) Escherichia coli cells with pGEX-4T-2 vector (GE Healthcare) and were purified. Brains of E18 rat embryos (either sex was used) were homogenized and lysed in the lysis buffer with $1 \mathrm{~mm}$ EDTA and $0.5 \mathrm{~mm}$ DTT. Then, the lysate was incubated for $1 \mathrm{~h}$ with GST-tagged proteins and glutathione-Sepharose $4 \mathrm{~B}$ beads (GE Healthcare). The beads were subsequently washed three times in the lysis buffer containing $1 \mathrm{~mm}$ EDTA and 0.5 mм DTT. Precipitates were separated by SDS-PAGE and detected by Western blot analysis.

Statistical analysis. All statistical analysis was performed using Student's $t$ test. Error bars in graphs represent the SD.

\section{Results}

\section{Polarized distribution of NGL-2 in hippocampal neurons}

To explore the role of NGL-2 in neuronal axon differentiation, their expression and distribution were examined in cultured hippocampal neurons from E18 rats. NGL-2 was detected during the polarization process (Fig. $1 A$ ). Immunostaining showed that NGL-2 was evenly distributed in all of the multiple undifferentiated minor neurites in stage 2 neurons (Fig. $1 B, C$ ). However, in axon differentiation stage 3 neurons, NGL-2 was more abundant at the tip of the longest neurite, which will become the axon (Fig. $1 B-D)$. Moreover, the relative NGL-2 levels at the tip of the developing axon was significantly higher than in other neurites (Fig. $1 E)$. Thus, the distribution of NGL-2 protein polarizes in the axon, as hippocampal neurons differentiate.

\section{NGL-2 regulates axon differentiation}

To study the function of NGL-2 in neuron differentiation, hippocampal neurons were transfected with constructs carrying EGFPtagged NGL-2 or EGFP alone (Fig. 2A), and were analyzed for their axon differentiation at 3 days in vitro (DIV3; Fig. $2 B, C$ ). It was concluded that the axons of neurons had completed differentiation based on the following two criteria: (1) length of at least $100 \mu \mathrm{m}$ or two times longer than other neurites; and (2) expression of the axonal marker Tau-1 (Guo et al., 2007). At DIV3 following the transfection with EGFP-NGL-2, $52.3 \pm 1.9 \%(n=120)$ of neurons displayed multiple (two or more) axon-like processes. As a comparison, only $5.6 \pm 1.1 \%$ of neurons transfected with EGFP alone exhibit multiple axons (Fig. 2C). These data suggest that the overexpression of NGL-2 increases axon formation in hippocampal neurons.

We next transfected constructs carrying NGL-2 shRNA or control shRNA in hippocampal neurons (Fig. 2D), and analyzed them for axon differentiation at DIV3 (Fig. $2 E, F$ ). In NGL-2-deficient neurons (as identified by GFP fluorescence), $55.5 \pm 3.2 \%(n=120)$ developed a single axon, $37.2 \pm 2.3 \%$ had no axons, and $7.3 \pm 2 \%$ exhibited multiple axons. In contrast, the majority of neurons transfected with control shRNA exhibited normal polarization, with a single axon in $80.1 \pm 3.5 \%(n=120)$ neurons, and no axon found in only $9.8 \pm 4.1 \%$ of neurons (Fig. $2 F$ ). To further confirm the effects of NGL-2 on axon differentiation, an RNAi-resistant NGL-2M was constructed (Fig. 2D). After hippocampal neurons were cotransfected with NGL-2 shRNA and NGL-2M, axon differentiation was observed at DIV3. As shown in Figure 2, E and F, compared with NGL-2 shRNA, NGL-2M significantly decreased the percentage of neurons without axons $(27.41 \pm 6 \%$ in neurons transfected with 1 $\mu \mathrm{g}$ of NGL-2M; $8.7 \pm 5.4 \%$ in neurons transfected with $3 \mu \mathrm{g}$ of NGL-2M) and increased the percentage of neurons with multiple axons $(9.8 \pm 4 \%$ in neurons transfected with $1 \mu \mathrm{g}$ of NGL-2M; $31.9 \pm 3.3 \%$ in neurons transfected with $3 \mu$ g of NGL-2M). These results indicate that NGL-2 promotes the differentiation of neurites into axons in hippocampal neurons.

\section{NGL-2 interacts with PAR complex by PAR6}

It has been demonstrated that PAR3/PAR6/aPKC $\zeta$ complex is required for axon differentiation (Shi et al., 2003; Chen et al., 2013). The colocalization of NGL-2 with PAR3 (Fig. 3A), PAR6 (Fig. 3B), or aPKC $\zeta$ (Fig. 3C) at the tip of developing axons of stage 3 hippocampal neurons, implies a potential NGL-2-PAR 
A

\section{DIV1 DIV2 DIV3 DIV4}

NGL-2

GAPDH

B
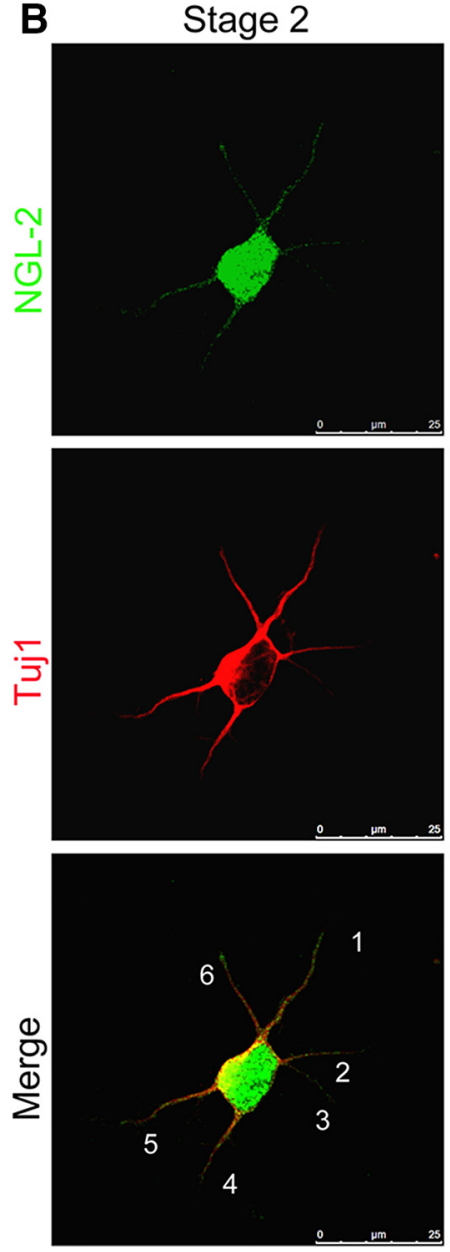
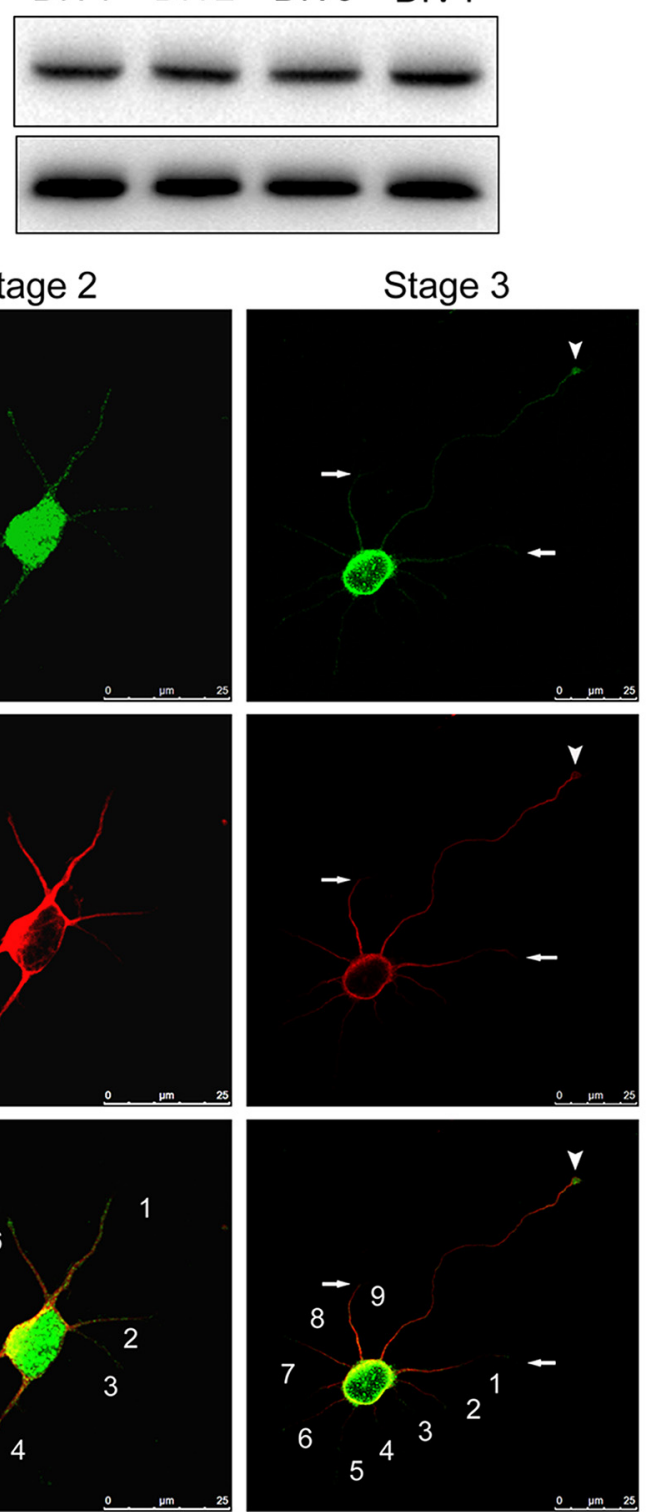
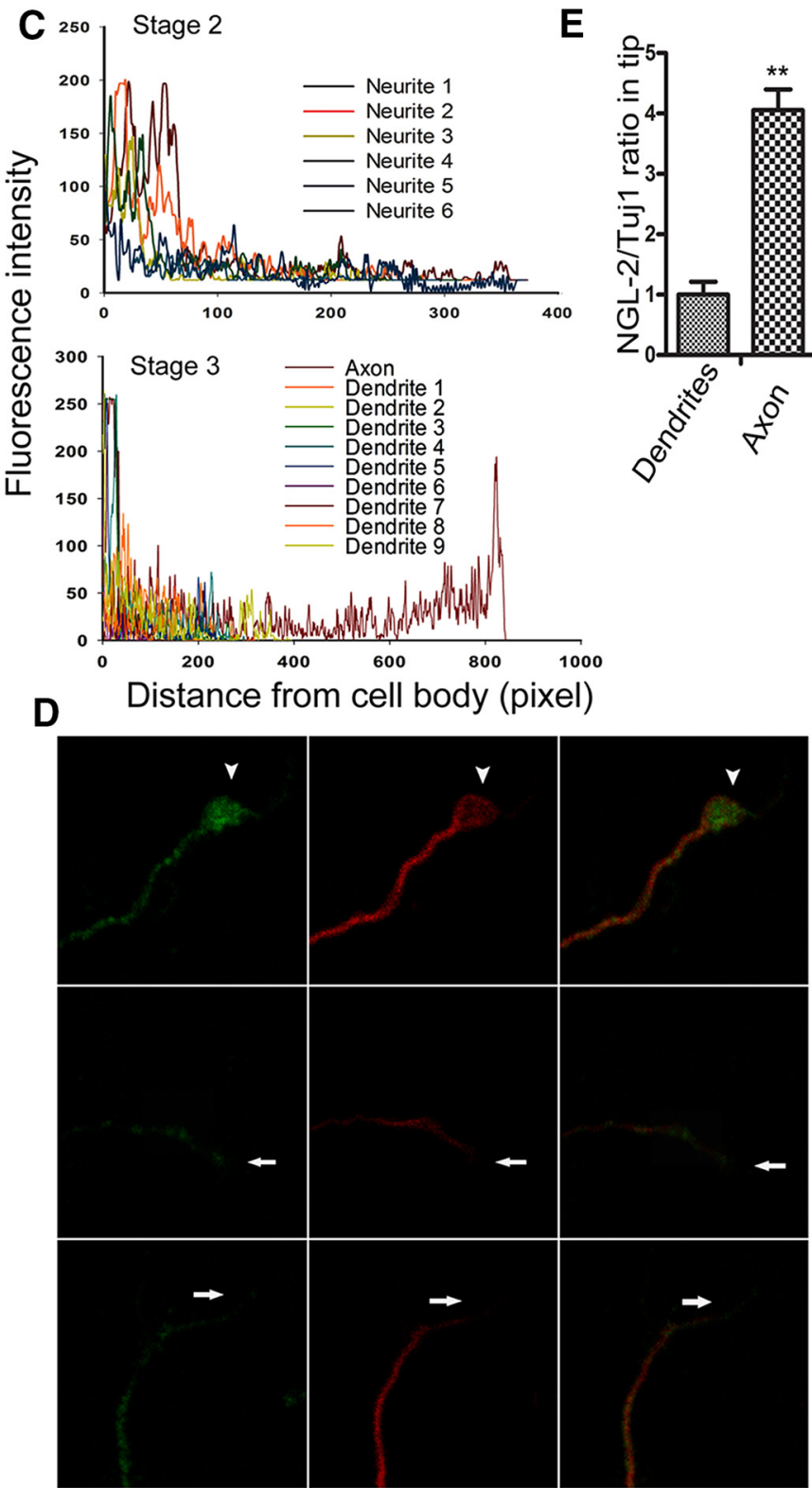

Figure 1. Distribution of NGL-2 in cultured hippocampal neurons. $A$, Expression of NGL-2 in primary neurons. Hippocampal neurons were isolated and cultured for different DIV. Cell lysates of primary neurons were subjected to immunoblotting with antibodies against NGL-2. B, Distribution of NGL-2 in stage 2 (left) and stage 3 (right) cultured hippocampal neurons. Hippocampal neurons at different stages were double stained with mouse anti-NGL-2 and rabbit anti-Tuj1 antibodies, which were visualized by FITC-conjugated goat anti-mouse antibody and rhodamine-conjugated anti-rabbit antibody, respectively. Arrowhead indicates the tip of axon. Arrow indicates the tip of dendrites. Scale bar, $25 \mu \mathrm{m}$. C, Profiles of NGL- 2 immunofluorescence intensity in the processes of stage 2 and stage 3 neurons. Fluorescence intensities were plotted against the distance from soma, as shown in $\boldsymbol{B}$. D. The higher-magnification pictures of stage 3 neurons, as shown in $\boldsymbol{B}$, right. Arrowhead indicates the tip of the axon. Arrow indicates the tip of dendrites. NGL-2 staining was more abundant at the tip of the axon than other neurites. E, NGL-2/TuJ1 ratios in neurite tips of stage 3 neurons. The NGL-2/TuJ1 ratio in dendrites was normalized to $1.00 \pm 0.11(n=50)$. The relative ratio in axons was $3.92 \pm 0.17(n=20)$. The error bars represent the SD. ${ }^{* *} p<0.01$, Student's $t$ test.

complex interaction. We then performed coimmunoprecipitation experiments with the homogenates from rat brains to test the possible interaction between NGL-2 and PAR3 (Fig. 3D,E), PAR6 (Fig. $3 F, G$ ), and aPKC $\zeta$ (Fig. $3 H, I$ ). In HEK293 cells transfected with Myc-tagged PAR6 and Flag-tagged NGL-2, these two proteins coimmunoprecipitated from the cell lysates, whereas Myc-tagged PAR3 or Myc-tagged aPKC $\zeta$ did not coimmunoprecipitate with Flag-tagged NGL-2 (Fig. 3J), suggesting that NGL-2 is incapable of directly interacting with PAR3 and aPKC $\zeta$. As PAR3, PAR6, and aPKC $\zeta$ are known to form the evolutionarily conserved PAR complex in mammals, NGL-2 may interact with PAR3 and aPKC $\zeta$ via PAR6. To test this possibility, HEK 293 cells were cotransfected with Myc-tagged PAR3, Flag- tagged NGL-2, and HA-tagged PAR6 or Myc-tagged aPKC $\zeta$, Flag-tagged NGL-2, and HA-tagged PAR6; the Myc-tagged PAR3 or Myc-tagged aPKC $\zeta$ was precipitated by Flag-tagged NGL-2 from the cell lysates (Fig. $3 K, L$ ). These results suggest that NGL-2, PAR3, PAR6, and aPKC $\zeta$ can form a complex, and PAR6 serves as a scaffold mediating the physical interaction of NGL-2 with PAR3 and aPKC $\zeta$.

\section{C terminus of NGL-2 binds to the PDZ domain of PAR6}

We next sought to determine the regions of PAR6 and NGL-2 responsible for their physical interaction. The extracellular region of NGL-2 contains nine LRRs, and a C2-type Ig domain. These domains are followed by a single transmembrane domain 

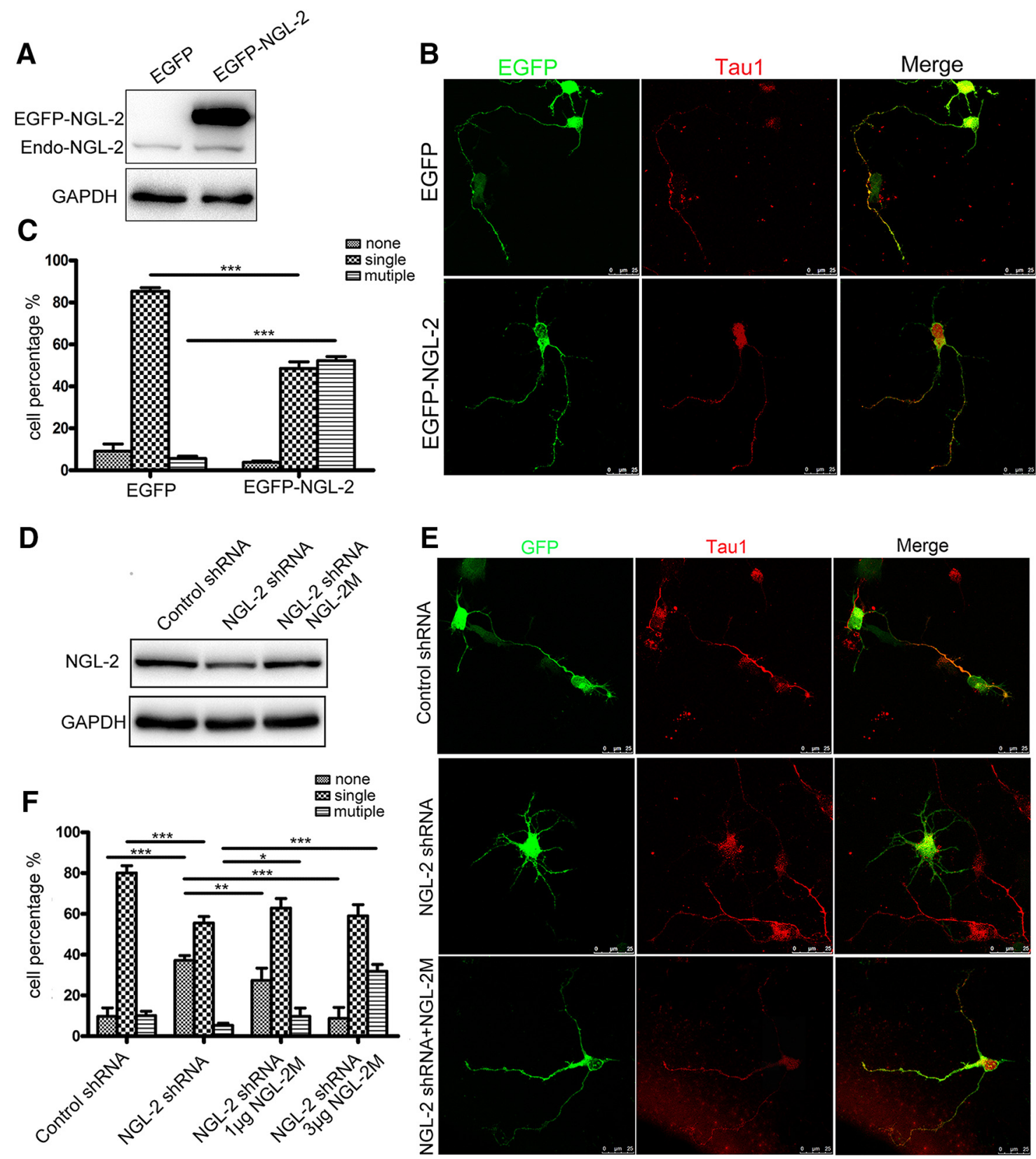

Figure 2. The effect of NGL-2 on axon differentiation. $A$, The transfection efficiency of NGL-2. Primary neurons were transfected with pEGFP-NGL-2 or pEGFP-empty vector. The level of NGL-2 was assessed by immunoblotting. $\boldsymbol{B}$, Increase in axon numbers in neurons overexpressing NGL-2. Hippocampal neurons were transfected with pEGFP-NGL-2 or pEGFP-empty vector. Transfected neurons were identified by EGFP and stained with axonal marker Tau1. Scale bar, $25 \mu \mathrm{m}$. C, Quantitative analysis of axon differentiation transfected by NGL-2. Transfected neurons were classified into the following three groups: none, neurons with short neurites in similar length; single, neurons with one process that was positive for Tau1 staining, and $>100 \mu \mathrm{m}$ and at least twice as long as the second longest process; and multiple, neurons with two or more processes positive for Tau 1 staining and $>100 \mu \mathrm{m}$ or twice as long as other neurites. The error bars represent the SD. ${ }^{* * *} p<0.001$, Student's $t$ test. $\boldsymbol{D}$, The efficiency of NGL-2 knockdown. Primary neurons were transfected with control shRNA, NGL-2 shRNA or cotransfected with an RNAi-resistant NGL-2M and NGL-2 shRNA. The level of NGL-2 was assessed by immunoblotting. $\boldsymbol{E}$, The axon formation transfected by NGL-2 shRNA. Hippocampal neurons transfected with control shRNA, NGL-2 shRNA or cotransfected with NGL-2M and NGL-2 shRNA were identified by GFP and cultured for $72 \mathrm{~h}$. Axons were identified by anti-Tau1 antibodies. Scale bar, $25 \mu \mathrm{m}$. F, Quantitative analysis of axon differentiation transfected by NGL-2 shRNA. The error bars represent the SD. ${ }^{*} p<0.05,{ }^{* *} p<0.01,{ }^{* * *} p<0.001$, Student's $t$ test.

and a cytoplasmic region, which ends with a PDZ binding domain (Kim et al., 2006; Fig. 4A). The PDZ binding domain is a specific domain consisting of Ser/Thr-X-Val/Leu/Ile at the C terminus. This domain binds to PDZ domain-containing molecules (Xia et al., 2003; Excoffon et al., 2004; Shano et al., 2008).

A series of GST fusion proteins with different fragments of NGL-2 were constructed (Fig. 4A), and pull-down assays were performed. The results showed that a PDZ binding domain of NGL-2 was essential for interacting with PAR6 (Fig. 4B,C). This region was therefore defined as the PAR6 binding domain of NGL-2. At the same time, we used various PAR6 deletion mutants to map the domains required for the interaction with NGL-2. PAR6 is a scaffolding protein that contains PDZ, PB1 (Phox Bem1), and semiCRIB (Cdc42 Rac interacting binding) domains (Fig. 4D; Aranda et al., 2008). GST pull-down assays showed that the PDZ domain interacted directly with NGL-2, but that PB1 or CRIB failed to bind NGL-2 (Fig. 4E, F). These data suggest that the C terminal of NGL-2 is physically associated with the PDZ domain of PAR6. 

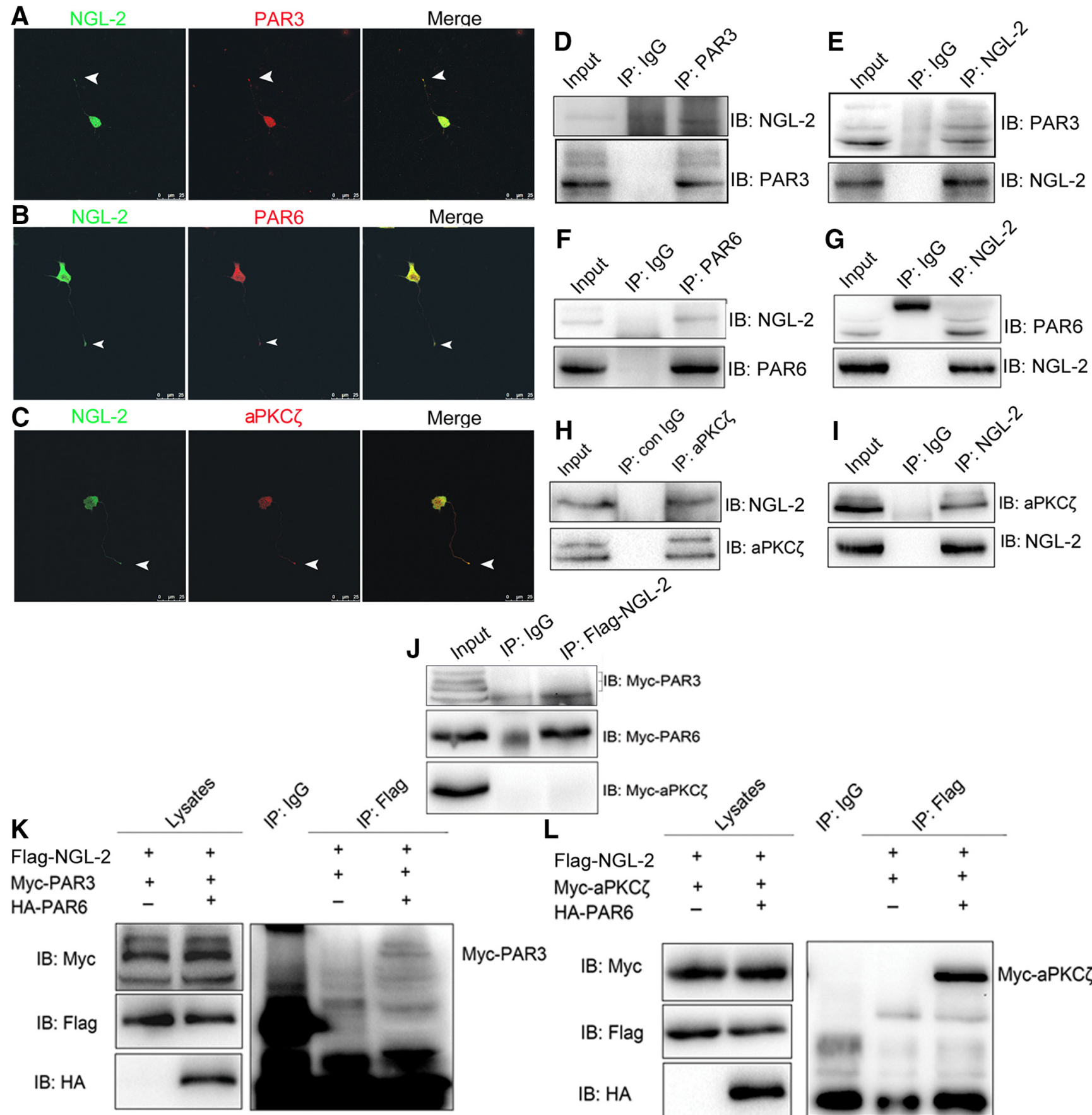

\section{IB: Myc-PAR3}

IB: Myc-PAR6
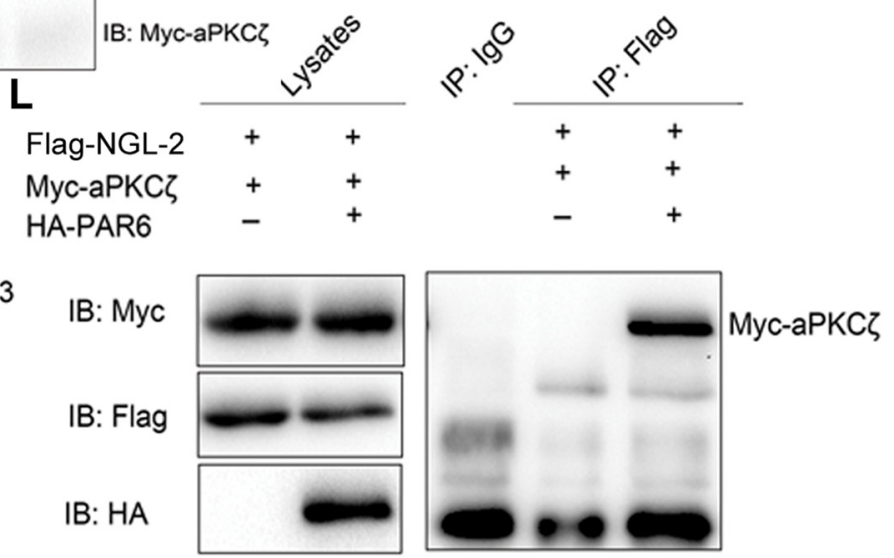

Figure 3. NGL-2 interacts with PAR complex by means of PAR6. A-C, Colocalization of NGL-2 and PAR complex in hippocampal neurons. A-C, Hippocampal neurons at stage 3 were double stained with anti-NGL-2 and anti-PAR3 $(\boldsymbol{A})$, anti-PAR6 $(\boldsymbol{B})$, or anti-aPKC $\zeta(\boldsymbol{C})$ antibodies. Arrowhead indicates the tip of the axon. Scale bar, $25 \mu \mathrm{m}$. $\boldsymbol{D}-\boldsymbol{I}$, Interaction between endogeneous NGL-2 and PAR complex proteins in rat brain; homogenates (500 $\mu$ g of protein) of E18 rat brains were incubated with antibodies against PAR3 (D), PAR6 (F), aPKC $\zeta(\boldsymbol{H})$, or NGL-2 (E, G, I). Control lgGs (con IgG) were used as controls. Resulting immunocomplexes were subjected to immunoblotting with indicated antibodies. Five percent of lysates (25 $\mu \mathrm{g}$ of protein) were loaded as input. J, Interaction between NGL-2 and PAR6, PAR3, or aPKC $\zeta$ proteins in HEK 293 cells. HEK 293 cell lysates expressing the indicated proteins were incubated with anti-Flag antibody. The bound proteins and coimmunoprecipitates were analyzed by immunoblotting with anti-Myc antibody. $\boldsymbol{K}$, Formation of NGL-2/PAR6/PAR3 ternary complex. HEK 293 cells were cotransfected with Flag-NGL-2 and Myc-PAR3 with or without HA-PAR6. Immunoprecipitates with anti-Flag antibody were subjected to immunoblotting with anti-Myc antibody. Lysates were also probed for the expression of Myc-PAR3, Flag-NGL-2, and HA-PAR6. L, Formation of NGL-2/PAR6/aPKC $\zeta$ ternary complex in HEK 293 cells. HEK 293 cells were cotransfected with Flag-NGL-2 and Myc-aPKC $\zeta$ with or without HA-PAR6. Immunoprecipitates with anti-Flag antibody were subjected to immunoblotting with anti-Myc antibody. Lysates were also probed for the expression of Myc-aPKC $\zeta$, Flag-NGL-2, and HA-PAR6.

\section{NGL-2 was recruited by PAR6 and polarized distribution at} the tip of axon

Having observed the physical interaction between NGL-2 and PAR6, we next asked whether such an association is necessary for the polarized distribution of NGL-2 in neurons. To test this, we inhibited PAR6 expression in hippocampal neurons by using
PAR6 siRNA. The expression and distribution of NGL-2 was examined in neurons by immunocytochemistry. As shown in Figure $5 A-C$, the polarized distribution of NGL-2 in neurons was significantly compromised after PAR6 inhibition.

To further confirm the involvement of PAR6 in the subcellular location of NGL-2, EGFP-tagged NGL-2 (EGFP-NGL-2) or 
A
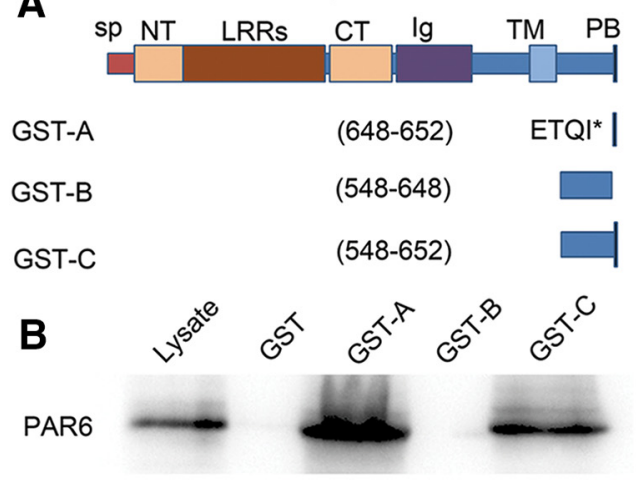

C

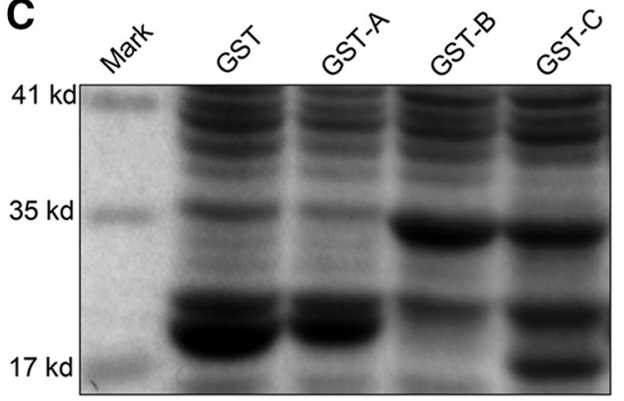

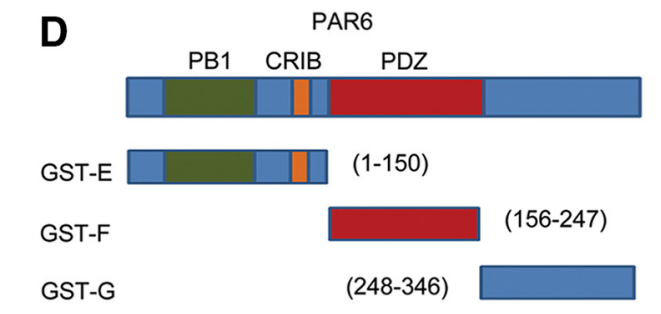

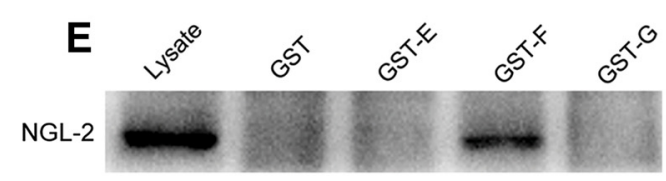

$\mathbf{F}$

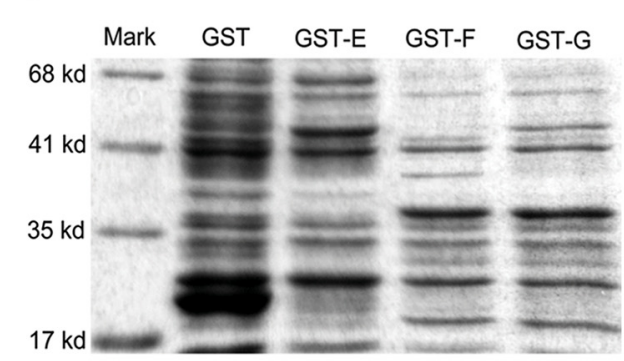

Figure 4. PDZ binding domain of NGL-2 binds to PDZ domain of PAR6. A, Domain structure and deletion constructs of NGL-2. SP, Signal peptide; NT, LRRN-terminal domain; CT, LRR C-terminal domain; Ig, Ig domain; TM, transmembrane domain; PB, PDZ domain binding domain; ETQ**, the last four amino acid residues of NGL-2. B, Mapping of the region in NGL-2 that is required for binding to PAR6. Lysate of E18 rat brain was incubated with NGL-2 deletions immobilized on glutathione-Sepharose beads. Bound proteins were subjected to immunoblotting with anti-PAR6 antibody. PDZ binding domain of NGL-2 was essential for pulling down PAR6. C, The GST fusion proteins used are indicated by Coomassie Brilliant Blue staining. D, Domain structure and deletion constructs of PAR6. PB1, Phox and Bem1; CRIB, Cdc42/Rac interactive binding; PDZ, PSD-95/Dlg/Z0-1. E, Mapping of the region in PAR6 that is required for binding to NGL-2. GST protein-immobilized beads were incubated with $\mathrm{E} 18$ rat brain lysate. The bound proteins were analyzed by immunoblotting with anti-NGL-2 antibody. Only the PDZ domain binds directly with NGL-2.F, The GST fusion proteins used are indicated by Coomassie Brilliant Blue staining.

DsRed-tagged PAR6 (DsRed-PAR6), respectively, was transfected in HEK 293 cells. EGFP-NGL-2 localized along the cytomembrane and showed nonpolarized distribution (Fig. 5D,E). However, DsRed-PAR6 was highly concentrated at one side of the cell and exhibited a polarized distribution (Fig. $5 F, G$ ). When HEK 293 cells were cotransfected with EGFP-NGL-2 and DsRed-PAR6, EGFP-NGL-2 protein colocalized with DsRed-PAR6 signals (Fig. $5 \mathrm{H}, \mathrm{I}$ ). The results suggested that the interaction of NGL-2 and PAR6 is critical for the subcellular distribution of NGL-2. Furthermore, in HEK 293 cells cotransfected with DsRed-PAR6 and EGFP-NGL-2 $\triangle \mathrm{PB}$ in which PDZ binding domain was deleted, EGFP-NGL-2 $\triangle \mathrm{PB}$ was distributed along the cytomembrane, distinct with the location of PAR6 (Fig. 5J,K). We also found that, compared with the polarized distribution of NGL-2 in normal neurons (Fig. $1 B$ ), EGFP-NGL-2 $\triangle \mathrm{PB}$ is not particularly abundant at the tip of an axon, but showed punctated distribution in nearly all neurites in both stage 2 and stage 3 neurons (Fig. 6A,B). Unlike neurons expressing EGFP-NGL-2 (Fig. 2B,C), axon differentiation was not affected by expression of EGFP-NGL-2 $\triangle \mathrm{PB}$ (Fig. $6 C, D$ ). These observations suggest that PAR6 recruits NGL-2 to exhibit the polarized subcellular distribution, and PDZ binding domain is an important domain for the association of NGL-2 with PAR6. In addition, the distribution of PAR3 and aPKC $\zeta$ was also examined, and NGL-2 did not significantly alter its subcellular localization in HEK 293 cells with the expression of DsRed-tagged PAR3 or DsRed-tagged aPKC $\zeta$ (data not shown).
NGL-2 controls the spatial polarization of stabilized microtubules to promote axon differentiation by aPKC ל/MARK2 signaling pathway

Previous studies (Witte et al., 2008; Chen et al., 2013) have shown that microtubules are preferentially stabilized in the designated future axon, and this polarized microtubule stability is both necessary and sufficient to drive axon differentiation. Consistent with this, we found that in polarizing hippocampal neurons expressing EGFP, the expression level of acetylated $\alpha$-tubulin, a mark of stabilized microtubules, was significantly higher in the emerging axon than other neurites (Fig. 7A,B). As shown in Figure 2E, NGL-2 overexpression promoted multiple axons. In neurons overexpressing EGFP-NGL-2, high levels of acetylated $\alpha$-tubulin were found in multiple neurites (Fig. $7 A, B$ ). Whereas, in neurons transfected with NGL-2 shRNA, the expression level of acetylated $\alpha$-tubulin was significantly lower in all neurites, and NGL-2-deficient neurons also failed to be polarized (Fig. 7C,D). These results suggest that, during neuron development, NGL-2 stabilizes microtubules and controls the spatial polarization of stabilized microtubules.

How does NGL-2 stabilize microtubules? NGL-2 activated $\mathrm{p}$-aPKC $\zeta$ in hippocampal neurons, but there was no effect on total aPKC $\zeta$. The activated p-aPKC $\zeta$ after NGL-2 overexpression was accompanied by an elevated phosphorylation of MARK2 at a specific site Thr 595, which was known to result in inhibition of MARK2 kinase activity and dephosphorylation of Tau. Conversely, knockdown of the endogenous NGL-2 resulted in a decrease of p-MARK2 and an increase of p-Tau (Fig. 8A,B). Dephosphorylated Tau could bind, bundle, and stabilize micro- 
A

A NGL-2

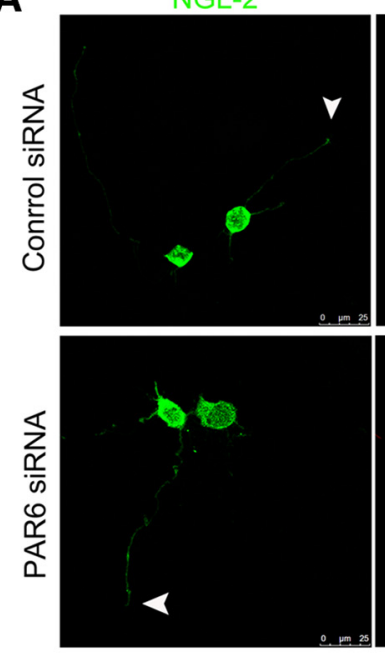

D

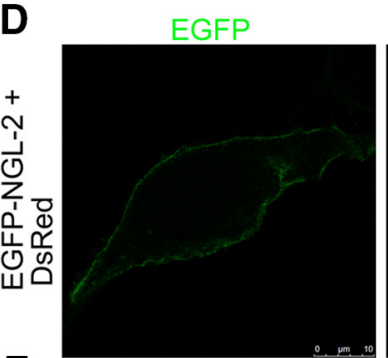

F
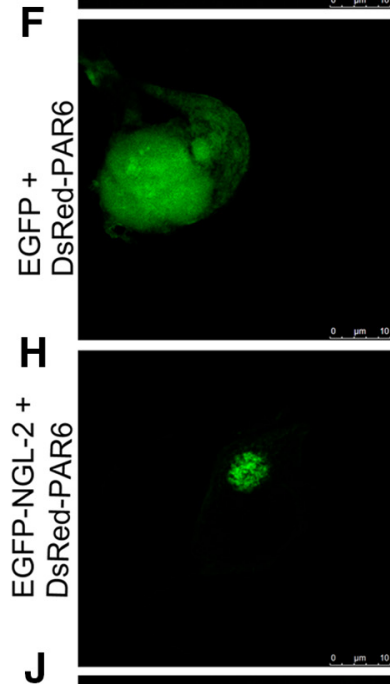

J

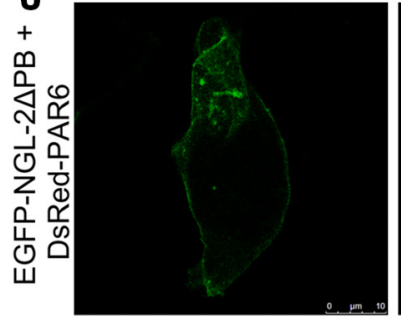

Tuj1
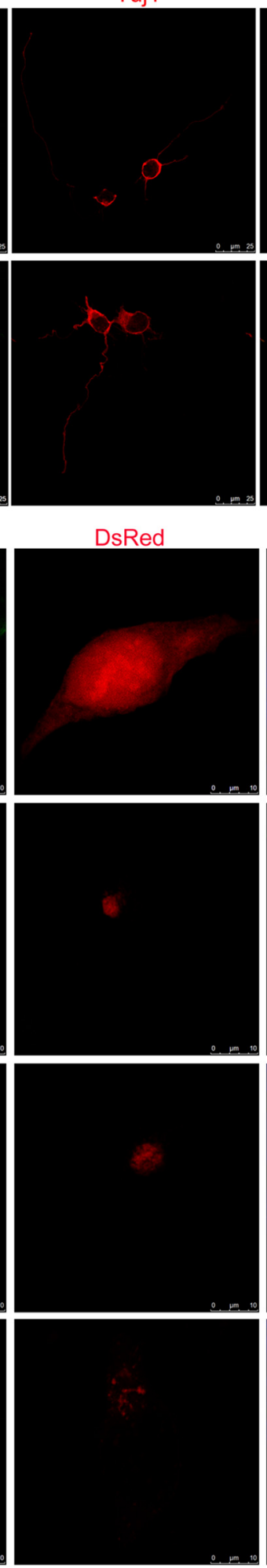

Merge
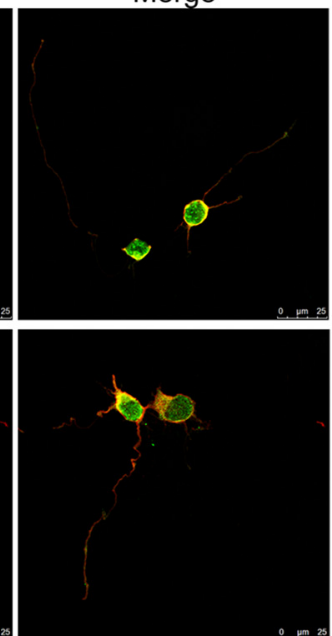

EGFP/DsRed/DAPI
B

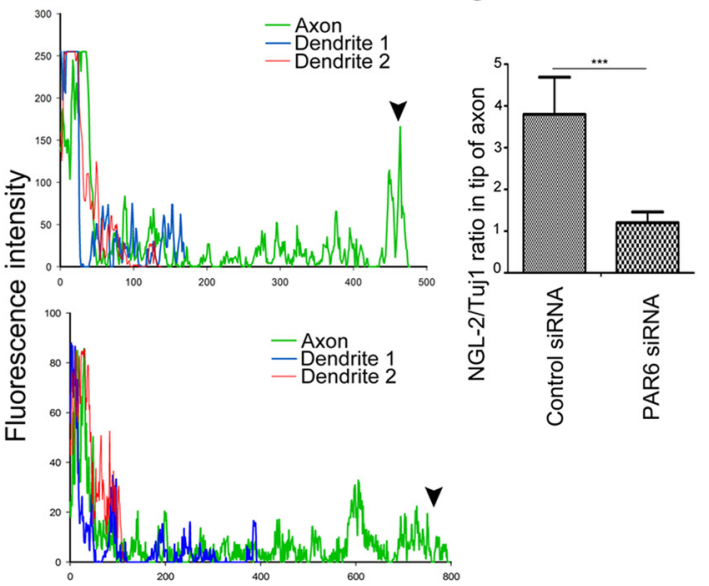

Distance from cell body (pixel)

E

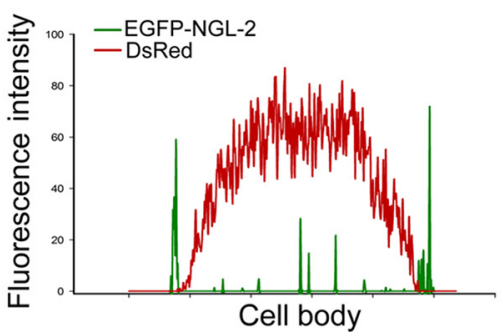

G
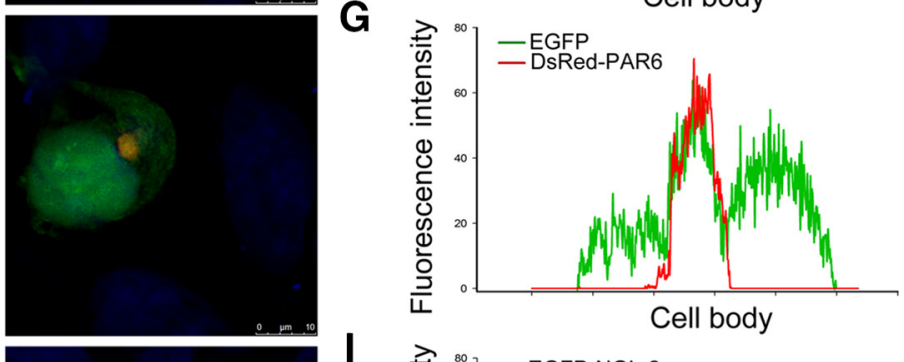

I

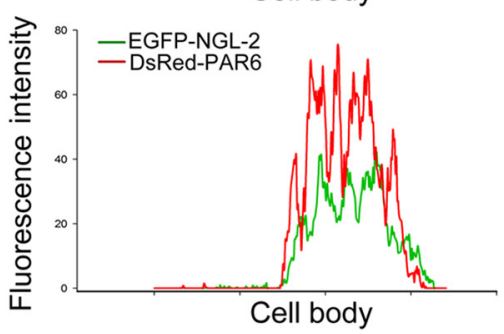

$\mathrm{K}$
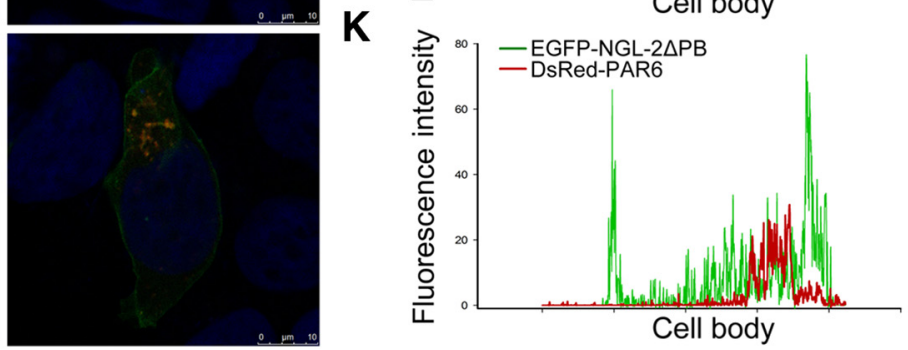

Figure 5. NGL-2 is recruited by PAR6 and polarized distribution at the tip of axon. $A$, The effect of the knockdown of PAR6 on NGL-2 distribution. Hippocampal neurons were transfected with PAR6 siRNA or control siRNA. Arrowhead indicates the tip of the axon. Scale bar, $25 \mu \mathrm{m}$. B, Profiles of NGL-2 immunofluorescence intensity in the processes of stage 2 and stage 3 neurons. Fluorescence intensities were plotted against the distance from soma, as shown in $A$. C, NGL-2/Tuj1 ratios in axon tips of stage 3 neurons. The NGL-2/Tuj1 ratio in the axon tip of neurons transfected with control siRNA was $3.81 \pm 0.89(n=50)$. The NGL-2/Tuj1 ratio in the axon tip of neurons transfected with PAR6 siRNA was $1.2 \pm 0.26(n=50)$. The error bars represent the SD. ${ }^{* * *} p<0.001$, Student's $t$ test. $D-I$, PAR6 recruits NGL-2 to one side of the HEK 293 cells. HEK 293 cells were transfected with EGFP-NGL-2 without or with DsRed-PAR6. After $48 \mathrm{~h}$, cells were fixed. Scale bar, $10 \mu \mathrm{m}$. The immun ofluorescence intensity of EGFP-NGL-2 and DsRed-PAR6 signals were measured by ImageJ. $\boldsymbol{J}, \boldsymbol{K}$, HEK 293 cells were cotransfected with EGFP-NGL-2 $\triangle \mathrm{PB}$ and DsRed-PAR6. After $48 \mathrm{~h}$, cells were fixed. Scale bar, $10 \mu \mathrm{m}$. The immunofluorescence intensity of NGL-2 and PAR6 signals was measured by ImageJ. 
A
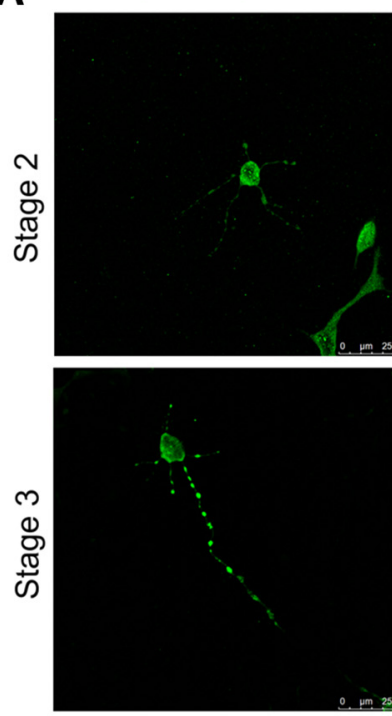

C
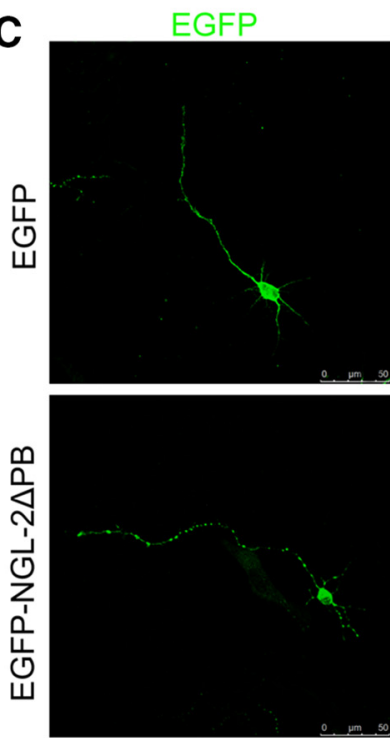

Tuj1
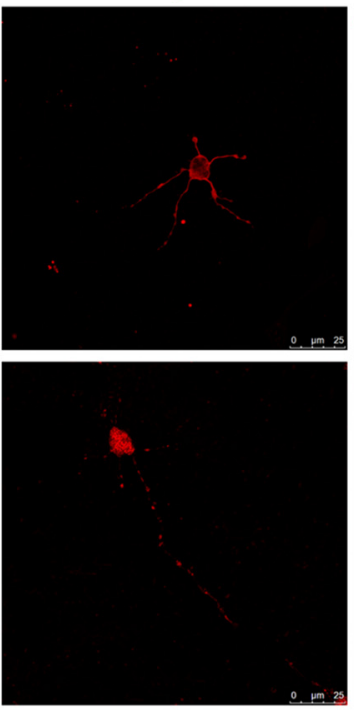

Tau-1
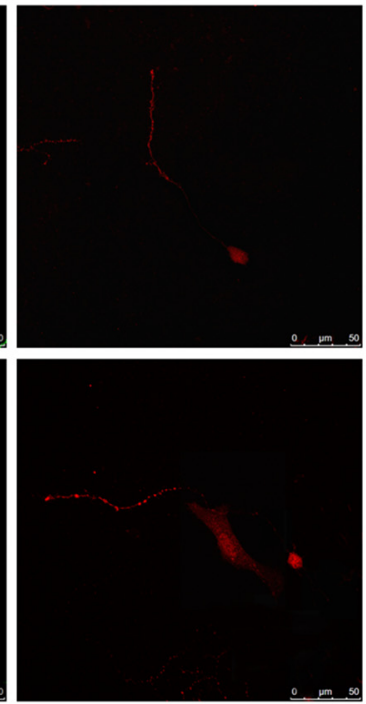

Merge
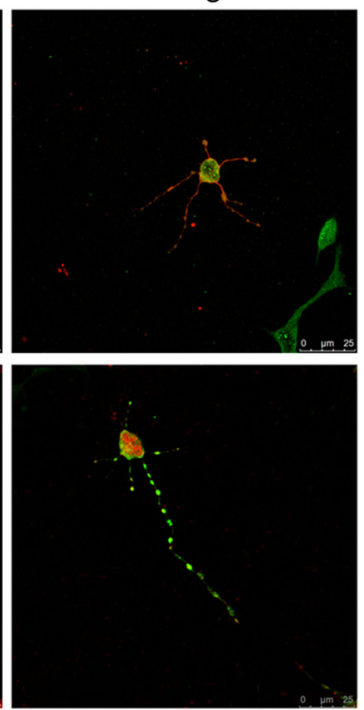

Merge
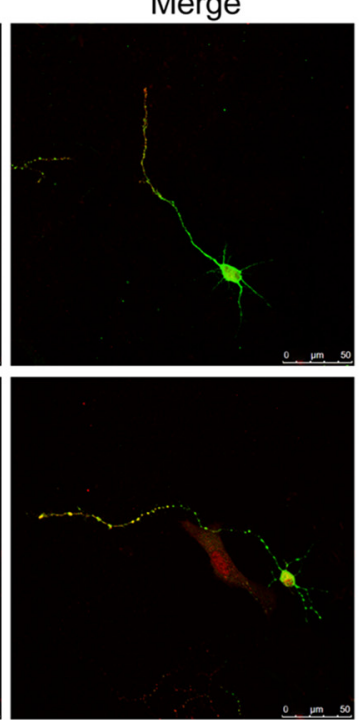

B

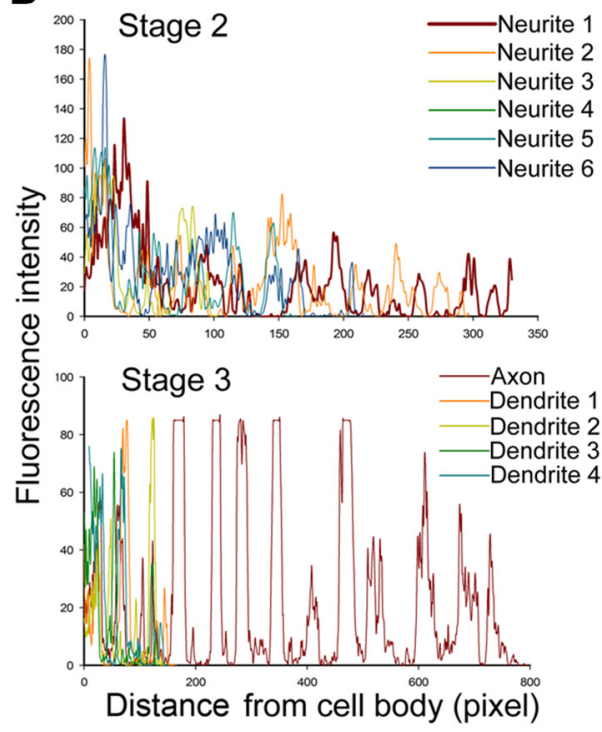

D

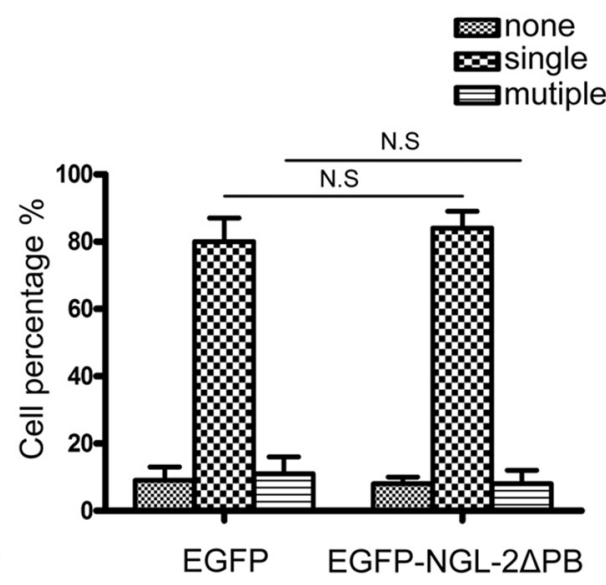

Figure 6. Distribution and function of NGL-2 $\triangle P B$ in hippocampal neurons. A, Hippocampal neurons were transfected with pEGFP-NGL-2 $\triangle \mathrm{PB}$. After $24 \mathrm{~h}$ (stage 2 ) or $48 \mathrm{~h}$ (stage 3 ), cells were fixed and stained with neuron marker Tuj1. Scale bar, $25 \mu \mathrm{m}$. B, Profiles of NGL-2 $\triangle \mathrm{PB}$ immunofluorescence intensity in the processes of stage 2 and stage 3 neurons. Fluorescence intensities were plotted against the distance from the soma, as shown in $A$. $C$, The effect of NGL-2 $\triangle \mathrm{PB}$ overexpression on axon differentiation. Hippocampal neurons were transfected with pEGFP-NGL-2 $\triangle \mathrm{PB}$ or pEGFP-empty vector. Transfected neurons were identified by EGFP and stained with axonal marker Tau1. Scale bar, $25 \mu \mathrm{m}$. D, Quantitative analysis of axon differentiation transfected by NGL-2 $\triangle$ PB. N.S., Not significant (Student's t test).

tubules, leading to the differentiation of the axon (Chen et al., 2006). The notion that NGL-2-driven differentiation is mediated by aPKC $\zeta$ was further supported by the finding that in neurons transfected with NGL-2 shRNA and DsRed-aPKC $\zeta, 73 \pm 2 \%$ $(n=40)$ developed axon-like processes positive for Taul. In contrast, in neurons transfected with NGL-2 shRNA and DsRed-PAR6, $40 \pm 5 \%(n=42)$ exhibited normal polarization (Fig. $8 C-F)$, suggesting that reduced axon differentiation resulting from the downregulation of NGL-2 could be prevented by aPKC $\zeta$, but not by PAR6. All of these results indicated that NGL-2 promotes axon differentiation via the aPKC $\zeta / M A R K 2$ signaling pathway.

\section{Discussion}

Neurons undergo different developmental stages during their polarization. This process follows well described morphological changes: neurons transform from round cells bearing lamellipo- dia (stage 1) into multipolar cells with several minor neurites (stage 2). At this stage, the neuron still has an unpolarized morphology. Neuronal symmetry breaks when one of the minor neurites extends rapidly to differentiate into an axon (stage 3 ), while the other neurites remain short and develop into dendrites at later stages (stage 4). This is followed by functional maturation, and the formation of dendritic spines and synapses (stage 5; Tahirovic and Bradke, 2009; Neukirchen and Bradke, 2011b). NGL-2 has been identified as a trans-synaptic adhesion molecule regulating the development and plasticity of synapses in hippocampal neurons (Kim et al., 2006; Woo et al., 2009; DeNardo et al., 2012; Soto et al., 2013). But in other stages, especially during axon differentiation, the function of NGL-2 is not well understood. Our study demonstrated that NGL-2 plays a critical role in axon differentiation in hippocampal neurons. Enhanced expres- 

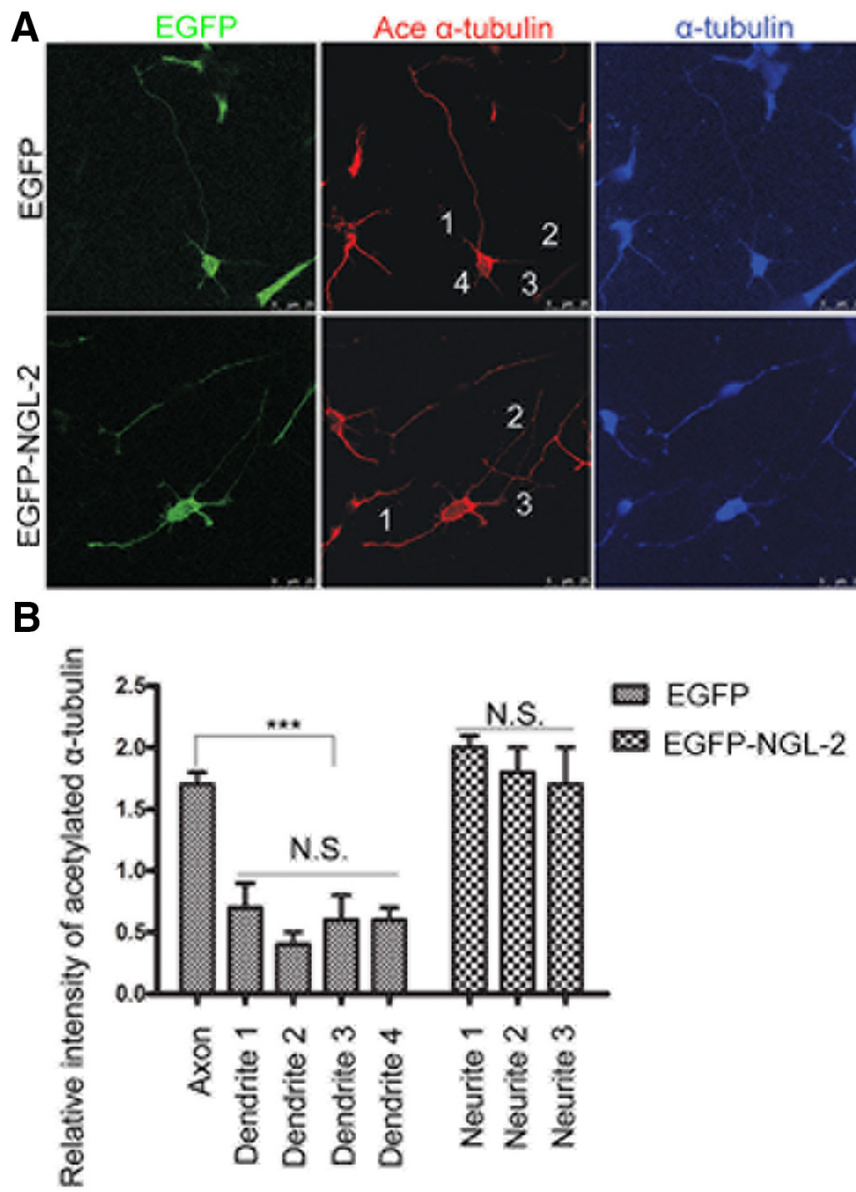
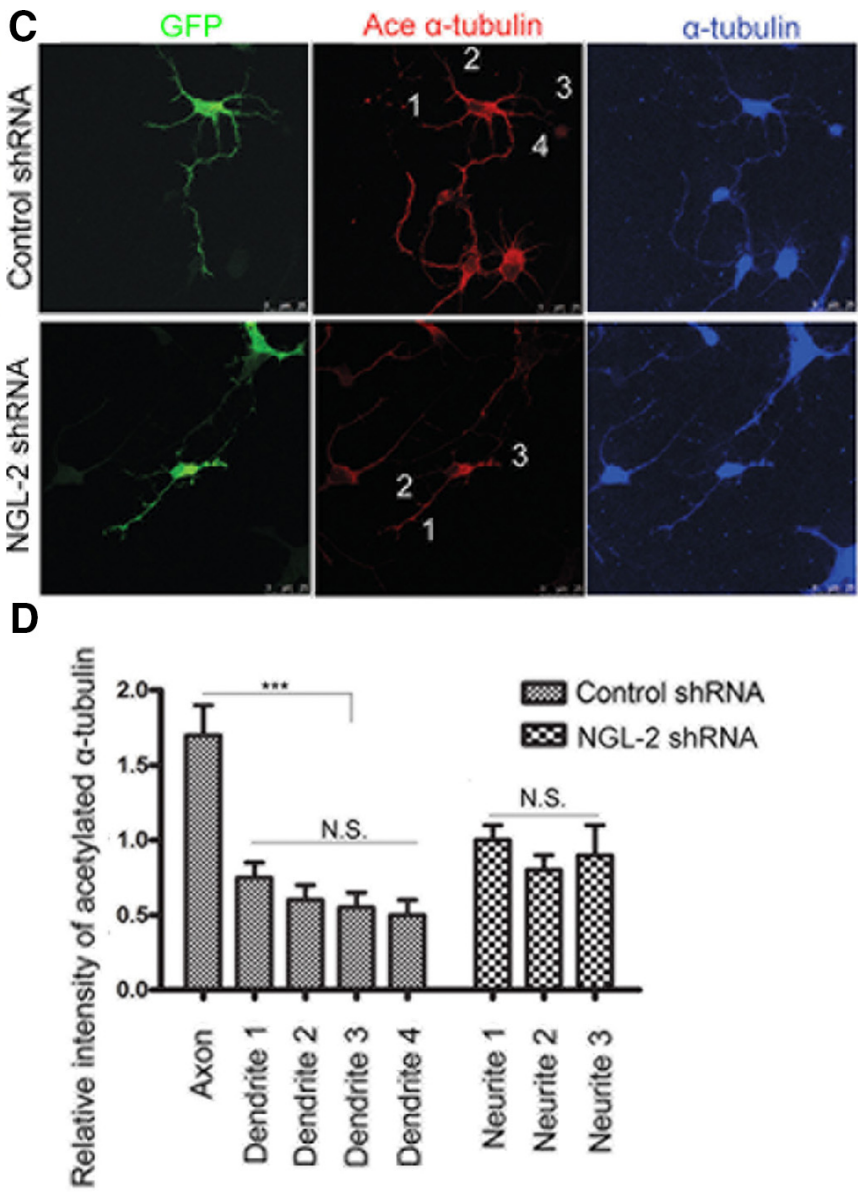

Figure 7. NGL-2 controls the spatial polarization of stabilized microtubules. $\boldsymbol{A}$, NGL-2 overexpression increased the relative intensity of acetylated $\alpha$-tubulin in multiple neurites of neurons. Hippocampal neurons were transfected with pEGFP-NGL-2 or pEGFP-empty vector. Transfected neurons were identified by EGFP. Cells were fixed and stained with anti-acetylated $\alpha$-tubulin antibodies, a stabilized microtubule marker, and anti- $\alpha$-tubulin. Scale bar, $25 \mu \mathrm{m}$. B, Quantification of the relative fluorescence intensity of acetylated $\alpha$-tubulin vs $\alpha$-tubulin. The error bars represent the SD. ${ }^{*} p<0.001$, Student's $t$ test. N.S., Not significant. C, NGL-2 shRNA decreased the relative fluorescence intensity of acetylated $\alpha$-tubulin in all neurites of neurons. Hippocampal neurons transfected with NGL-2 shRNA or control shRNA were identified by GFP. Cells were fixed and stained with anti-acetylated $\alpha$-tubulin antibodies and anti- $\alpha$-tubulin. Scale bar, $25 \mu$ m. $D$, Quantification of the relative intensity of acetylated $\alpha$-tubulin vs $\alpha$-tubulin. The error bars represent the SD. * $p<0.001$, Student's $t$ test. N.S., Not significant.

sion of NGL-2 promotes axon formation in neurons, whereas knockdown of NGL-2 blocks the axon formation and neuron polarization. The function of NGL-2 in neurons relies on the interaction with PAR complex, which is mediated by PAR6.

PAR complex of PAR3, PAR6, and atypical PKC has emerged as a central player in the mechanisms that regulate cell polarity in the different cell types of various organisms ranging from worms to mammals (Aranda et al., 2008). Many binding partners for this complex have been implicated in regulating the polarity of cells, such as the small GTPase Cdc42 (Joberty et al., 2000), the kinesin motor protein KIF3A (Nishimura et al., 2004), the guanine exchange factor Tiam1/STEF (Nishimura et al., 2005), the lipid and protein phosphatase PTEN (Feng et al., 2008), the GTPase activating protein (GAP) p190RhoGAP (Zhang and Macara, 2008), the tumor suppressor lethal giant larvae (Lgl) (Plant et al., 2003), the ubiquitin ligases Smurf1 and Smurf2 (Schwamborn et al., 2007), and the transforming growth factor receptor 1 (Ozdamar et al., 2005). For the first time, we have demonstrated that NGL-2 can interact with PAR complex. Such a physical association with PAR complex is necessary for the polarized subcellular distribution of NGL-2, as well as the axon-promoting capacity of NGL-2.

Endogenous NGL-2 was coprecipitated with PAR3, PAR6, and $\mathrm{aPKC} \zeta$, respectively, in the homogenates from the rat brain. But, in fact, the coprecipitation phenomena between NGL-2 and
PAR3 or NGL-2 and aPKC $\zeta$ cannot be detected in HEK 293 cells except by the presence of PAR6. These results suggested that PAR6 is the essential element among the components of the complex to interact with NGL-2, and NGL-2 binds directly to PAR6, which is required for the interaction of NGL-2 with PAR3 and aPKC $\zeta$. In our results, the polarity accumulation of PAR6 recruited NGL-2 to the same sites in both HEK 293 cells and hippocampal neurons. Knockdown of PAR6 expression disturbed the polarity distribution of NGL-2. Therefore, polarized distribution of NGL-2 depends on the presence of PAR6. Previous studies showed that the evolutionarily conserved NGLs proteins share the same PDZ binding domain in their $\mathrm{C}$ terminal, which interacts with the first two PDZ domains of PSD-95 protein, and are interdependent with PSD-95 for synaptic localization (Kim et al., 2006). We found that the PDZ binding domain of NGL-2 bound the PDZ domain of PAR6. Deletion of the PDZ binding domain also disturbs the polarity distribution of NGL-2. These results further demonstrate that NGL-2 first binds to PAR6, then is recruited by PAR6 to be involved in the axon differentiation, and the PDZ binding domain is the critical domain for the polarized distribution of NGL-2. In the presence of PAR6, NGL-2 interacts with PAR3 and aPKC $\zeta$ in HEK 293 cells and brain.

Over the past decade, PAR6 has emerged as a key player in a plethora of cellular polarity processes in organisms ranging from 
A

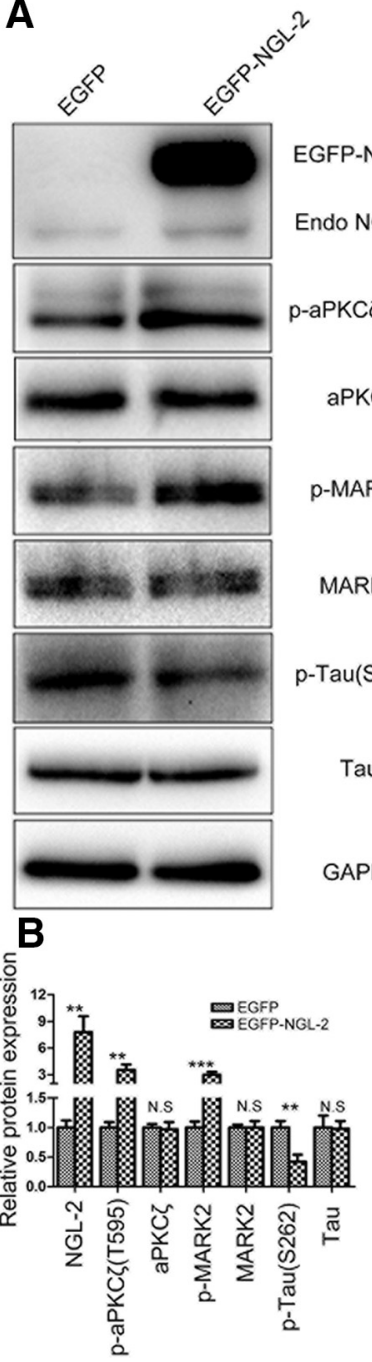

E

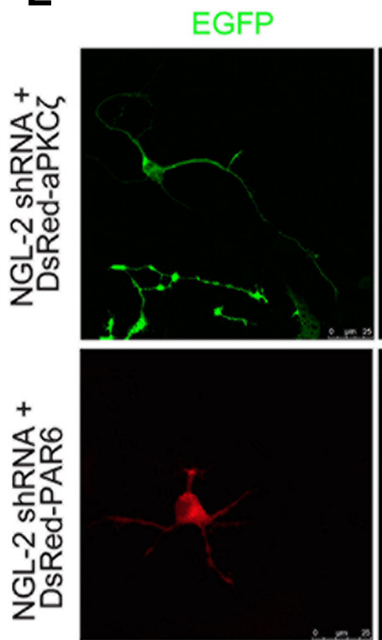

C
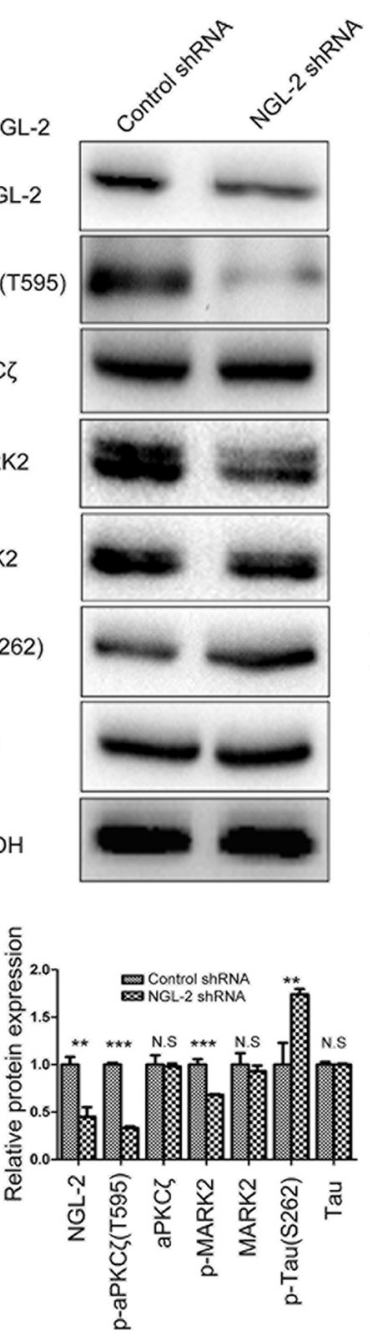

D
Tau-1
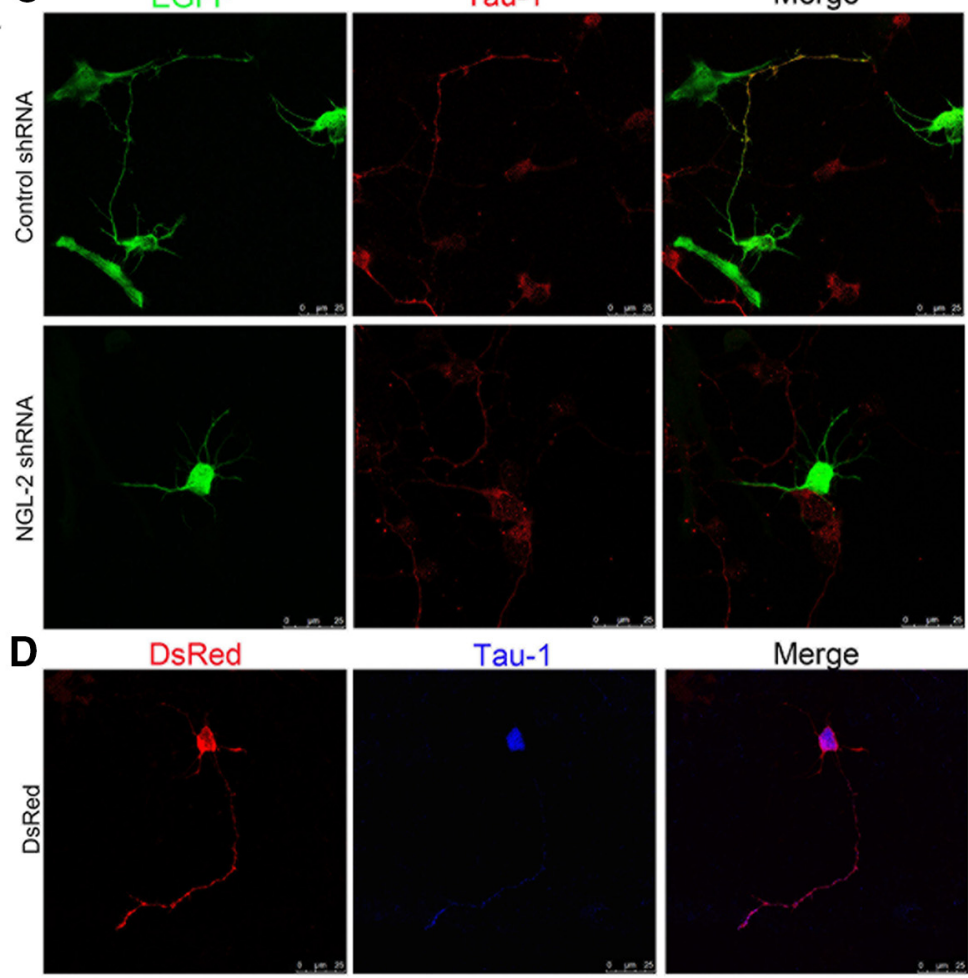

Tau-1
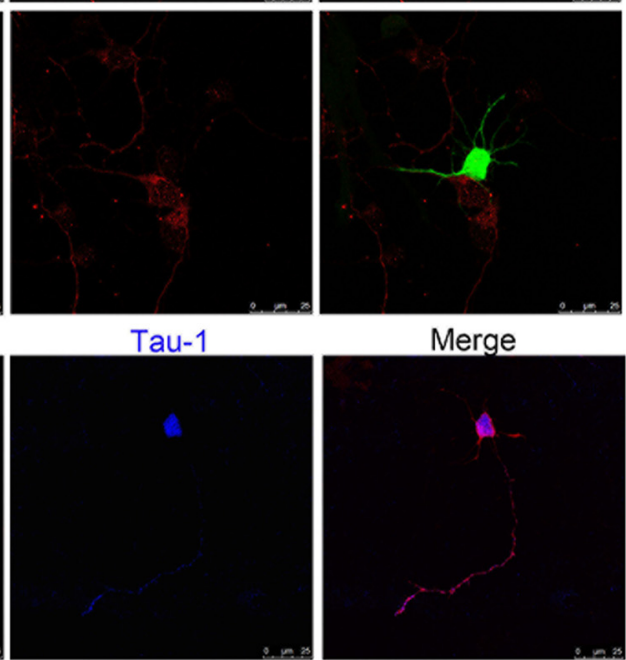

Merge
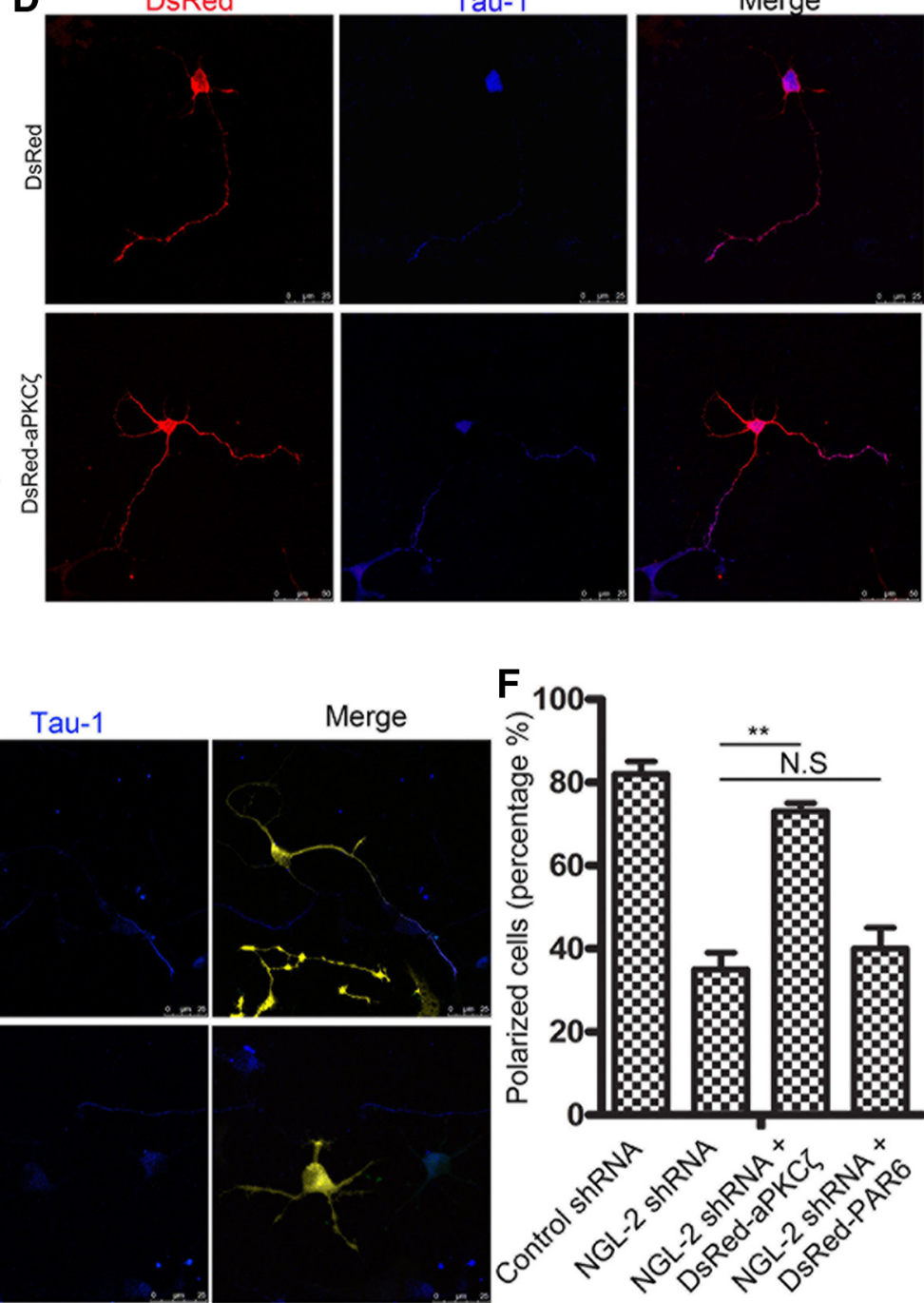

Figure 8. NGL-2 promotes axon differentiation by the aPKC $\zeta / M A R K 2$ signaling pathway. $\boldsymbol{A}, \boldsymbol{B}, \mathrm{NGL}-2$ regulates tau phosphorylation by aPKC $\zeta /$ MARK2 singaling pathway. $\boldsymbol{A}$, Hippocampal neurons transfected by pEGFP-NGL-2 (left) or NGL-2 shRNA (right) were lysed and analyzed by Western blotting with indicated antibodies. Blotting with anti-GAPDH antibody showed equal amount of loading. $\boldsymbol{B}$, The relative protein expression was normalized to GAPDH. The error bars represent the SD of three experiments. ${ }^{* *} p<0.01$, ${ }^{* * *} p<0.001$, Student's $t$ test. N.S., Not significant. $\boldsymbol{C}-\boldsymbol{E}$, Overexpression of aPKC $\zeta$ counteracts the polarity defect caused by NGL-2 suppression. Hippocampal neurons cotransfected with NGL-2 shRNA and DsRed-aPKC $\zeta$ or DsRed-PAR6 were identified by GFP and DsRed. Axons were identified by anti-Tau1 antibodies. Scale bar, $25 \mu \mathrm{m}$. $\boldsymbol{F}$, Quantification of the percentage of neurons that are polarized with a single axon [control shRNA, $82 \pm 3 \%$ ( $n=$ 40); NGL-2 shRNA, $35 \pm 4 \%(n=40) ;$ NGL-2 shRNA + DsRed-aPKC $\zeta, 73 \pm 2 \%(n=40)$; NGL-2 shRNA + DsRed-PAR6, $40 \pm 5 \%(n=42)]$. The error bars represent the SD. ${ }^{*} p<0.001$, Student's $t$ test. N.S., Not significant. 
worms to humans (Henrique and Schweisguth, 2003). PAR6 is a hub and embedded in an extensively interconnected network of polarity complex interactions, such as PAR6-PAR3-Cdc42-aPKC complex and PAR6-Lgl-Scrb complex (Bose and Wrana, 2006). Our unpublished data identified that $\mathrm{Cdc} 42$ was also a protein that interacted with NGL-2 by a liquid chromatography-tandem mass spectrometry shotgun analysis and coimmunoprecipitation. So, the spatial conformation of PAR6 protein provided an interaction platform for PAR3, NGL-2, Cdc42, and aPKC to communicate, share information, and collaborate.

Initial neuronal polarization has been described as a "tug of war" in which each neurite aims to become the axon (Craig and Banker, 1994). In this model, an intracellular re-enhancing feedback loop singles out one neurite to become the axon, while intrinsic inhibitory cues repress the growth of the other neurites (Neukirchen and Bradke, 2011a). In recent years, it has been shown that the microtubule cytoskeleton could be part of this proposed positive feedback loop in the future axon (Neukirchen and Bradke, 2011a). Microtubules exhibit a higher stability along the shaft of axons than do minor neurites. Consistently, local and transient microtubule stabilization of one minor neurite stimulates its maturation into an axon (Witte et al., 2008). Furthermore, modest pharmacological microtubule stabilization promotes the establishment of supernumerary axons (Neukirchen and Bradke, 2011b).

Recent studies (Shi et al., 2003; Chen et al., 2013) reported that the PAR complex is required for axon differentiation. PAR3/ PAR6/aPKC $\zeta$ were found in multiple undifferentiated minor processes of nonpolarized stage 2 hippocampal neurons and were abundant in the axon, being particularly concentrated at its tip in polarized stage 3 hippocampal neurons (Shi et al., 2003). aPKC $\zeta$ negatively regulates MARK2 activity to prevent Tau phosphorylation and thereby to maintain the stability of microtubules in axons (Chen et al., 2006). Our results showed that, similar to PAR3/PAR6/aPKC $\zeta$, NGL-2 was evenly distributed in all of the multiple undifferentiated minor neurites in stage 2 neurons. NGL-2 was more abundant at the tip of the axon only in stage 3 neurons. Overexpression of NGL-2 in hippocampal neurons led to increased levels of $\mathrm{p}$-aPKC $\zeta$. Conversely, knockdown of the endogenous NGL-2 resulted in a decrease of p-aPKC $\zeta$. These findings led us to focus the microtubule stability regulation mechanism of NGL-2 on the interaction between NGL-2 and aPKC $\zeta$. The increased level of $\mathrm{p}-\mathrm{aPKC} \zeta$ following NGL-2 overexpression was accompanied with elevated phosphorylation of MARK2 and dephosphorylation of Tau. Moreover, the axon differentiation defect caused by the knockdown of NGL-2 was rescued by aPKC $\zeta$ overexpression. In contrast, PAR6 overexpression cannot prevent the phenotype. These observations suggested that NGL-2 regulates microtubule stability and axon differentiation by activating the aPKC $/$ /MARK2 signaling pathway. From the above, endogenous NGL-2 is an enhancer of the intracellular re-enhancing feedback loop in normal hippocampal neuronal polarization. The polarized accumulation of NGL-2 in single neurites increased the local stabilization of microtubules and made it a priority for growth in an axon. As we described in Materials and Methods, exogenous NGL-2 was transfected into hippocampal neurons before being plated onto coverslips. This means that the effect of exogenous NGL-2 overexpression has worked in stage 2 neurons. The interaction between exogenous NGL-2 and endogenous PAR3/PAR6/aPKC $\zeta$ complex increased the stabilization of microtubules in multiple undifferentiated minor neurites in stage 2 neurons and broke the normal feedback loop, promoting the establishment of multiple axons. This notion has been proven by an RNAi-resistant NGL-2 rescue exper- iment. An shRNA-resistant NGL-2M not only recovered the percentage of normal neurons with a single axon but also increased the percentage of neurons with multiple axons.

Our study demonstrates that PAR6 is responsible for the polarized distribution of NGL-2 in the emerging axon. After being recruited by PAR6, NGL-2 regulates microtubule stability to control axon differentiation by regulating $\mathrm{aPKC} \zeta$. The binding to PAR6 and the subsequent activation of aPKC $\zeta$ is critical for the function of NGL-2 in axon differentiation.

\section{References}

Aranda V, Nolan ME, Muthuswamy SK (2008) Par complex in cancer: a regulator of normal cell polarity joins the dark side. Oncogene 27:68786887. CrossRef Medline

Bose R, Wrana JL (2006) Regulation of Par6 by extracellular signals. Curr Opin Cell Biol 18:206-212. CrossRef Medline

Chen S, Chen J, Shi H, Wei M, Castaneda-Castellanos DR, Bultje RS, Pei X, Kriegstein AR, Zhang M, Shi SH (2013) Regulation of microtubule stability and organization by mammalian Par3 in specifying neuronal polarity. Dev Cell 24:26-40. CrossRef Medline

Chen YM, Wang QJ, Hu HS, Yu PC, Zhu J, Drewes G, Piwnica-Worms H, Luo ZG (2006) Microtubule affinity-regulating kinase 2 functions downstream of the PAR-3/PAR-6/atypical PKC complex in regulating hippocampal neuronal polarity. Proc Natl Acad Sci U S A 103:8534-8539. CrossRef Medline

Craig AM, Banker G (1994) Neuronal polarity. Annu Rev Neurosci 17:267310. CrossRef Medline

DeNardo LA, de Wit J, Otto-Hitt S, Ghosh A (2012) NGL-2 regulates inputspecific synapse development in CA1 pyramidal neurons. Neuron 76 : 762-775. CrossRef Medline

Excoffon KJ, Hruska-Hageman A, Klotz M, Traver GL, Zabner J (2004) A role for the PDZ-binding domain of the coxsackie B virus and adenovirus receptor (CAR) in cell adhesion and growth. J Cell Sci 117:4401-4409. CrossRef Medline

Feng W, Wu H, Chan LN, Zhang M (2008) Par-3-mediated junctional localization of the lipid phosphatase PTEN is required for cell polarity establishment. J Biol Chem 283:23440-23449. CrossRef Medline

Guo W, Jiang H, Gray V, Dedhar S, Rao Y (2007) Role of the integrin-linked kinase (ILK) in determining neuronal polarity. Dev Biol 306:457-468. CrossRef Medline

Henrique D, Schweisguth F (2003) Cell polarity: the ups and downs of the Par6/aPKC complex. Curr Opin Genet Dev 13:341-350. CrossRef Medline

Joberty G, Petersen C, Gao L, Macara IG (2000) The cell-polarity protein Par6 links Par3 and atypical protein kinase C to Cdc42. Nat Cell Biol 2:531-539. CrossRef Medline

Kim S, Burette A, Chung HS, Kwon SK, Woo J, Lee HW, Kim K, Kim H, Weinberg RJ, Kim E (2006) NGL family PSD-95-interacting adhesion molecules regulate excitatory synapse formation. Nat Neurosci 9:12941301. CrossRef Medline

Lin D, Edwards AS, Fawcett JP, Mbamalu G, Scott JD, Pawson T (2000) A mammalian PAR-3-PAR-6 complex implicated in Cdc42/Racl and aPKC signalling and cell polarity. Nat Cell Biol 2:540-547. CrossRef Medline

Neukirchen D, Bradke F (2011a) Cytoplasmic linker proteins regulate neuronal polarization through microtubule and growth cone dynamics. J Neurosci 31:1528-1538. CrossRef Medline

Neukirchen D, Bradke F (2011b) Neuronal polarization and the cytoskeleton. Semin Cell Dev Biol 22:825-833. CrossRef Medline

Nishimura T, Kato K, Yamaguchi T, Fukata Y, Ohno S, Kaibuchi K (2004) Role of the PAR-3-KIF3 complex in the establishment of neuronal polarity. Nat Cell Biol 6:328-334. CrossRef Medline

Nishimura T, Yamaguchi T, Kato K, Yoshizawa M, Nabeshima Y, Ohno S, Hoshino M, Kaibuchi K (2005) PAR-6-PAR-3 mediates Cdc42-induced Rac activation through the Rac GEFs STEF/Tiam1. Nat Cell Biol 7:270277. CrossRef Medline

Noda Y, Takeya R, Ohno S, Naito S, Ito T, Sumimoto H (2001) Human homologues of the Caenorhabditis elegans cell polarity protein PAR6 as an adaptor that links the small GTPases Rac and Cdc42 to atypical protein kinase C. Genes Cells 6:107-119. CrossRef Medline

Ozdamar B, Bose R, Barrios-Rodiles M, Wang HR, Zhang Y, Wrana JL (2005) Regulation of the polarity protein Par6 by TGFbeta receptors 
controls epithelial cell plasticity. Science 307:1603-1609. CrossRef Medline

Plant PJ, Fawcett JP, Lin DC, Holdorf AD, Binns K, Kulkarni S, Pawson T (2003) A polarity complex of mPar-6 and atypical PKC binds, phosphorylates and regulates mammalian Lgl. Nat Cell Biol 5:301-308. CrossRef Medline

Schwamborn JC, Khazaei MR, Püschel AW (2007) The interaction of $\mathrm{mPar} 3$ with the ubiquitin ligase Smurf2 is required for the establishment of neuronal polarity. J Biol Chem 282:35259-35268. CrossRef Medline

Shano S, Hatanaka K, Ninose S, Moriyama R, Tsujiuchi T, Fukushima N (2008) A lysophosphatidic acid receptor lacking the PDZ-binding domain is constitutively active and stimulates cell proliferation. Biochim Biophys Acta 1783:748-759. CrossRef Medline

Sheng M, Sala C (2001) PDZ domains and the organization of supramolecular complexes. Annu Rev Neurosci 24:1-29. CrossRef Medline

Shi SH, Jan LY, Jan YN (2003) Hippocampal neuronal polarity specified by spatially localized mPar3/mPar6 and PI 3-kinase activity. Cell 112:63-75. CrossRef Medline

Soto F, Watkins KL, Johnson RE, Schottler F, Kerschensteiner D (2013) NGL-2 regulates pathway-specific neurite growth and lamination, synapse formation, and signal transmission in the retina. J Neurosci 33: 11949-11959. CrossRef Medline

Stiess M, Bradke F (2011) Neuronal polarization: the cytoskeleton leads the way. Dev Neurobiol 71:430-444. CrossRef Medline

Tahirovic S, Bradke F (2009) Neuronal polarity. Cold Spring Harb Perspect Biol 1:a1644. CrossRef Medline
Tang H, Liu X, Wang Z, She X, Zeng X, Deng M, Liao Q, Guo X, Wang R, Li X, Zeng F, Wu M, Li G (2011) Interaction of hsa-miR-381 and glioma suppressor LRRC4 is involved in glioma growth. Brain Res 1390:21-32. CrossRef Medline

Tang H, Wang Z, Liu X, Liu Q, Xu G, Li G, Wu M (2012) LRRC4 inhibits glioma cell growth and invasion through a miR-185-dependent pathway. Curr Cancer Drug Targets 12:1032-1042. CrossRef Medline

Wiggin GR, Fawcett JP, Pawson T (2005) Polarity proteins in axon specification and synaptogenesis. Dev Cell 8:803-816. CrossRef Medline

Witte H, Neukirchen D, Bradke F (2008) Microtubule stabilization specifies initial neuronal polarization. J Cell Biol 180:619-632. CrossRef Medline

Woo J, Kwon SK, Kim E (2009) The NGL family of leucine-rich repeatcontaining synaptic adhesion molecules. Mol Cell Neurosci 42:1-10. CrossRef Medline

Wu M, Huang C, Li X, Li X, Gan K, Chen Q, Tang Y, Tang K, Shen S, Li G (2008) LRRC4 inhibits glioblastoma cell proliferation, migration, and angiogenesis by downregulating pleiotropic cytokine expression and responses. J Cell Physiol 214:65-74. CrossRef Medline

Xia Z, Hufeisen SJ, Gray JA, Roth BL (2003) The PDZ-binding domain is essential for the dendritic targeting of 5-HT2A serotonin receptors in cortical pyramidal neurons in vitro. Neuroscience 122:907-920. CrossRef Medline

Zhang H, Macara IG (2008) The PAR-6 polarity protein regulates dendritic spine morphogenesis through p190 RhoGAP and the Rho GTPase. Dev Cell 14:216-226. CrossRef Medline 